\title{
Influence of X-ray irradiation on the properties of the Hamamatsu silicon photomultiplier S10362-11-050C
}

\author{
Chen $\mathrm{Xu}^{a, *}$, Robert Klanner ${ }^{b}$, Erika Garutti ${ }^{b}$, Wolf-Lukas Hellweg ${ }^{b}$ \\ a DESY, Hamburg, Germany \\ ${ }^{b}$ Institute for Experimental Physics, University of Hamburg, Hamburg, Germany
}

\begin{abstract}
We have investigated the effects of X-ray irradiation to doses of 0, $200 \mathrm{~Gy}, 20 \mathrm{kGy}, 2 \mathrm{MGy}$, and 20 MGy on the Hamamatsu silicon-photomultiplier (SiPM) S10362-11-050C. The SiPMs were irradiated without applied bias voltage. From current-voltage, capacitance/conductancevoltage, capacitance/conductance-frequency, pulse-shape, and pulse-area measurements, the SiPM characteristics below and above breakdown voltage were determined. Significant changes of some SiPM parameters are observed. Up to a dose of $20 \mathrm{kGy}$ the performance of the SiPMs is hardly affected by X-ray radiation damage. For doses of 2 and 20 MGy the SiPMs operate with hardly any change in gain, but with a significant increase in dark-count rate and cross-talk probability.
\end{abstract}

Keywords: XFEL, silicon photomultipliers, MPPC, GAPD, X-ray radiation damage.

\section{Contents}

1 Introduction

2 X-ray radiation damage in silicon sensors 1

3 Sensors, Measurements, Analysis and Results 2

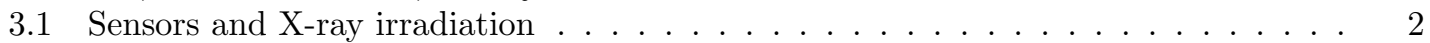

3.2 Equivalent model for SiPMs . . . . . . . . . . . . . . . . . 3

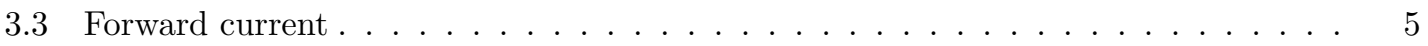

3.4 Reverse current . . . . . . . . . . . . . . . . . . . . . . . . . 7

3.5 Capacitance and conductance versus frequency . . . . . . . . . . . 8

3.6 Capacitance versus voltage and doping profile . . . . . . . . . . . . . 11

3.7 Gain and breakdown voltage . . . . . . . . . . . . . . . . . 13

3.8 Dark-count rate and dark current . . . . . . . . . . . . . . . . . . . . [15

3.9 Cross Talk . . . . . . . . . . . . . . . . . . . . . . . . . . . . . . . . . . . . . . . .

3.10 Pulse Shape . . . . . . . . . . . . . . . . . . . . . . . 16

4 Discussion of the results 18

5 Conclusions and Outlook 19

6 List of References $\quad 20$

${ }^{*}$ Corresponding author. Email address: Chen.Xu@desy.de, Telephone: +49 4089982964. 


\section{Introduction}

After more than 30 years of development, silicon-photomultipliers (SiPMs) are now well established high-gain photodetectors [1, 2], which already have found numerous applications [3]. A SiPM consists of a matrix of avalanche photodiodes connected in parallel and operated above the breakdown voltage in Geiger mode. Relevant parameters which characterize the SiPM performance are: signal shape, gain, dark-count rate, cross talk, afterpulse rate, breakdown voltage, and their dependencies on voltage and temperature.

As SiPMs detect single charge carriers, radiation damage is a major concern. In numerous investigations [4, 5] it has been found, that for high-energy radiation the dominant radiation effect for SiPMs is an increase in the dark-count rate due to defects in the silicon crystal. Given that radiation damage presents a serious limitation for many applications, several groups together with the producers of SiPMs are undertaking major efforts to make SiPMs more radiation tolerant.

In contrast to radiation-induced bulk damage, little is known on the effects on SiPMs of surface damage caused by X-rays and ionizing radiation. The authors of Ref. [6] have irradiated a prototype SiPM from Hamamatsu (Type No. T2K-11-100C) under bias up to $240 \mathrm{~Gy}$ of ${ }^{60} \mathrm{Co} \gamma$-rays and measured dark current, dark-count rate, gain, and cross talk. Whereas gain and cross talk did not significantly change with dose, large dark-count pulses and localized spots with leakage current along the edge of the active region and the bias lines were observed for about half an hour after X-ray irradiation for doses above $200 \mathrm{~Gy}$. As far as we know this study has not been pursued further. In Ref. [1] it is reported, that several SiPMs have been irradiated up to 500 Gy by a ${ }^{60} \mathrm{Co}$-source without applying a bias voltage during irradiation. No evidence for large pulses has been found after the irradiation. The authors of Ref. [7] have irradiated green-sensitive SiPMs (SENSL SSPM-0701BG-TO18) with $14 \mathrm{MeV}$ electrons to fluences between $3.1 \cdot 10^{7}$ and $3.8 \cdot 10^{8} \mathrm{~cm}^{-2}$ and observed a large increase in dark-count rate and a decrease in effective gain. In Ref. [8], in which the radiation hardness of Hamamatsu SiPMs was investigated, footnote 1 states: "An early irradiation test on SiPMs using a series of high activity ${ }^{137}$ Cs-sources in Jefferson Lab showed that SiPMs are insensitive to electromagnetic radiation and there was no significant change in performance of SiPMs up to 2 krad of gamma irradiation."

This paper first gives a short summary of X-ray radiation effects in silicon sensors, describes the methods used to determine the parameters of the Hamamatsu SiPMs using measurements below and above breakdown voltage, and finally presents the results for doses of 0, $200 \mathrm{~Gy}, 20 \mathrm{kGy}$, $2 \mathrm{MGy}$, and $20 \mathrm{MGy}$ of X-ray irradiation without applied bias voltage. Details of the measurements can also be found in Refs [9, 10. As we anticipate that X-ray radiation damage depends on the details of the SiPM design, we plan to extend these studies to SiPMs from other producers.

\section{X-ray radiation damage in silicon sensors}

$\mathrm{X}$-rays with energies below $300 \mathrm{keV}$, which is the threshold energy for the formation of defects in the silicon bulk, generate only defects in the dielectrics, at the $\mathrm{Si}-\mathrm{SiO}_{2}$ interface and at the interfaces between dielectrics. The effects of X-ray radiation damage are discussed in detail in Refs [1], 12. Here, we only give a short summary.

In $\mathrm{SiO}_{2}, \mathrm{X}$-rays produce on average one electron-hole $(e h)$ pair every $18 \mathrm{eV}$ of deposited energy. Depending on ionization density and electric field, a fraction of the $e h$ pairs recombine. The remaining charge carriers move in the $\mathrm{SiO}_{2}$ by diffusion and, if an electric field is present, by drift. Most electrons, due to their high mobility and relatively low trapping probability, leave the $\mathrm{SiO}_{2}$. However holes, which move via polaron hopping, are typically captured by deep traps in the $\mathrm{SiO}_{2}$ or at the $\mathrm{Si}-\mathrm{SiO}_{2}$ interface, which results in fixed positive charge states and interface traps. We denote the density of oxide charges by $N_{o x}$, and the density of the $\mathrm{Si}-\mathrm{SiO}_{2}$ interface traps by $N_{i t}$. The interface traps, if exposed to an electric field, act as generation centers for a surface current with density $J_{\text {surf }}$.

Results on $N_{o x}$ and $J_{\text {surf }}$ from MOS-Capacitors and Gate-Controlled-Diodes produced by different vendors and for different crystal orientations for X-ray doses between $10 \mathrm{kGy}$ and 1 GGy can be found in Refs [13 15]. For a dose of $10 \mathrm{kGy}$ the values for $N_{o x}$ are between $0.4 \cdot 10^{12}$ and 
$1.2 \cdot 10^{12} \mathrm{~cm}^{-2}$, and for $J_{\text {surf }}$ between 0.1 and $1 \mu \mathrm{A} / \mathrm{cm}^{2}$ at room temperature. Depending on technology and crystal orientation for doses of the order of 1 MGy the values of $N_{\text {ox }}$ and $J_{\text {surf }}$ saturate at $1.5-3.5 \cdot 10^{12} \mathrm{~cm}^{-2}$ and $2-6 \mu \mathrm{A} / \mathrm{cm}^{2}$, respectively. Before irradiation typical values are a few $10^{10} \mathrm{~cm}^{-2}$ and a few $\mathrm{nA} / \mathrm{cm}^{2}$, respectively. We note that in addition to differences due to technology, the values of $N_{o x}$ and of $J_{\text {surf }}$ at a given dose depend on the value and the orientation of the electric field in the oxide, and that there are significant annealing effects [14, 15].

The depleted $\mathrm{Si}-\mathrm{SiO}_{2}$-interface areas generate surface currents, and therefore we expect a significant increase in dark current below the breakdown voltage. In case a fraction of the charge carriers from the surface current reaches the amplification region, an increase in dark-count rate will also occur above the breakdown voltage. This however depends on the details of the SiPM design.

\section{Sensors, Measurements, Analysis and Results}

\subsection{Sensors and X-ray irradiation}

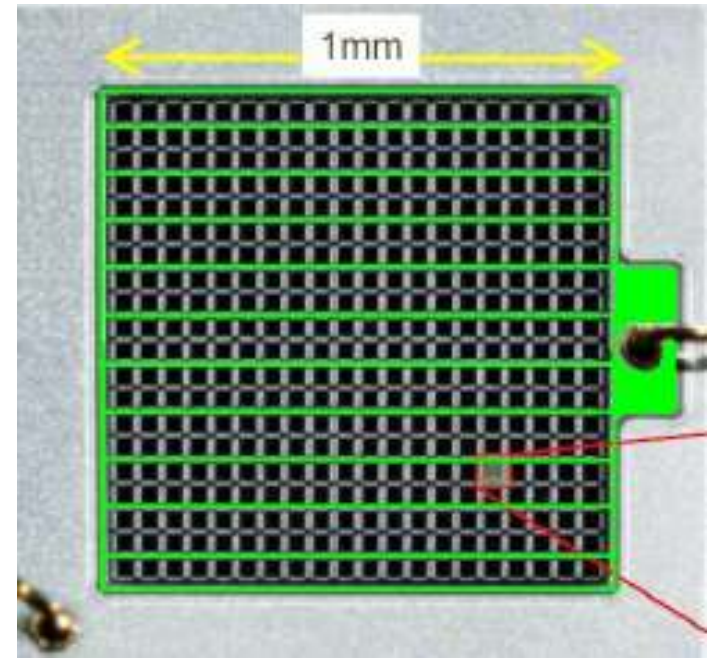

Fig. 1: Photo of the Hamamatsu S10362-11-050C taken from Ref. [1]. One can see $20 \times 20$ pixels, the biasing contact on the right, which is connected via the biasing lines to the individual pixels, and the readout contact on the bottom left. The pixel size is $50 \mu \mathrm{m} \times 50 \mu \mathrm{m}$.

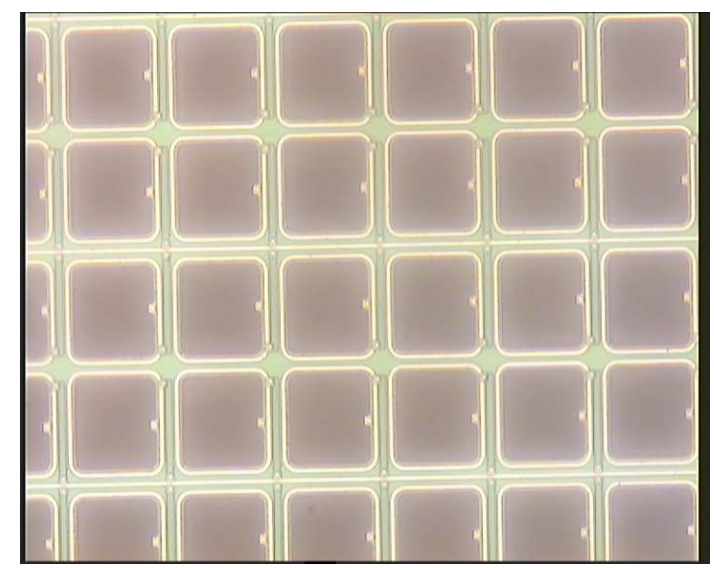

Fig. 2: Photo of the pixels of the Hamamatsu SiPM S10362-11-050C. The pixel size is $50 \mu \mathrm{m} \times 50 \mu \mathrm{m}$. 


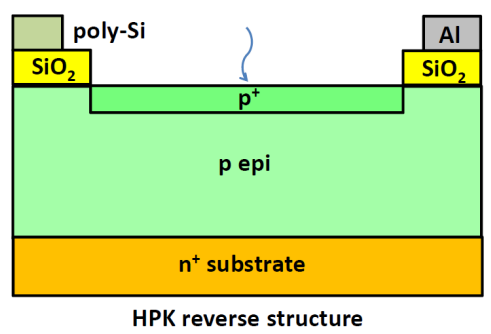

Fig. 3: Schematic cross-section of the Hamamatsu S10362-11-050C after Ref. 18. Compared to the figure given there, the $\mathrm{SiO}_{2}$ layer, the Al-contact line, and the poly-Si layer of the quenching resistor have been added. From the capacitance measured above full depletion we estimate a depth of the $p$-epitaxial layer of about $2.3 \mu \mathrm{m}$. We assume that the $p^{+}$implant is covered by an anti-reflection coating, which however should not affect the electrical properties of the SiPM.

Sensors of the type Hamamatsu S10362-11-050C [16] were used for the studies. They have 400 pixels of $50 \mu \mathrm{m} \times 50 \mu \mathrm{m}$ and a total area of $1 \mathrm{~mm} \times 1 \mathrm{~mm}$. Fig. 1 1 shows an overall view of the SiPM, Fig.2 details of the pixel region, and Fig. 3 a schematic cross-section. The biasing contact, which can be seen at the middle right of Fig. 1 is connected to the biasing lines which run horizontally between alternate pixel rows. Fig. 2 shows how the biasing lines are connected to the quench resistors which run up and down in between the pixels. The ends of the quench resistors are connected to square $\mathrm{Al}$ rings surrounding the pixels. The connections to the $p^{+}$implants are seen as the square dots at the middle right of every pixel. The readout contact to the $n^{+}$substrate is made from the top side of the SiPM via the bulk. The corresponding Al layer (silvery area) surrounds the entire pixel area, with a contact seen at the lower left corner of Fig.1.

The $\mathrm{Si}-\mathrm{SiO}_{2}$ interface areas are sensitive to X-ray radiation damage. These and their respective areas, estimated from the photographs, are:

- the region below the $\mathrm{Al}$ biasing ring which surrounds the entire pixel area: $4 \times 1 \mathrm{~mm} \times 38 \mu \mathrm{m}=$ $15.2 \cdot 10^{-4} \mathrm{~cm}^{2}$,

- the region below the $\mathrm{Al}$ biasing lines in between the pixels: $10 \times 1 \mathrm{~mm} \times 12 \mu \mathrm{m}=12 \cdot 10^{-4} \mathrm{~cm}^{2}$,

- the region below the quench resistors: $400 \times 40 \mu \mathrm{m} \times 12 \mu \mathrm{m}=19.2 \cdot 10^{-4} \mathrm{~cm}^{2}$, and

- the region in between the pixels not covered by the Al biasing line $9 \times 1 \mathrm{~mm} \times 10 \mu \mathrm{m}=$ $9 \cdot 10^{-4} \mathrm{~cm}^{2}$.

The total $\mathrm{Si}_{-} \mathrm{SiO}_{2}$-interface area is about $6 \cdot 10^{-3} \mathrm{~cm}^{2}$, and assuming a saturation value of $J_{\text {surf }}$ of $4 \mu \mathrm{A} / \mathrm{cm}^{2}$, an upper limit for the surface-generation current of $25 \mathrm{nA}$ can be estimated.

The X-ray irradiations up to $20 \mathrm{kGy}$ were performed at an X-ray tube (PW 2273/20 from PANalytical). Using a Mo target the dose rate in $\mathrm{SiO}_{2}$ at a distance of $20 \mathrm{~cm}$ was approximately $0.6 \mathrm{~Gy} / \mathrm{s}$. After characterizing the SiPMs, four have been irradiated to $200 \mathrm{~Gy}$ and two of those later to $20 \mathrm{kGy}$. No bias has been applied to the SiPM during irradiation. The X-ray irradiations to 2 MGy and 20 MGy were performed with X-rays of $8 \mathrm{keV}$ in the P11 beam line of PETRA III 17. with a dose rate of approximately $2 \mathrm{kGy} / \mathrm{s}$. Two sensors were irradiated to $2 \mathrm{MGy}$, and two others to $20 \mathrm{MGy}$. All irradiations and measurements were performed at $22-25^{\circ} \mathrm{C}$. In between irradiations and measurements the SiPMs were stored at $-20^{\circ} \mathrm{C}$ to prevent annealing.

\subsection{Equivalent model for SiPMs}

In order to characterize the SiPM, the $R C$ model shown in Fig.4 has been used, which is similar to the ones reported in Refs. 19 22. Our model, however, includes more elements, which allows us to check which of them are actually relevant and can be determined by the measurements. The left side shows a pixel with a Geiger discharge, the middle the remaining $N_{p i x}-1$ pixels without discharge followed by the parasitic capacitance, $C_{\text {par }}$, to describe the coupling of the biasing lines 


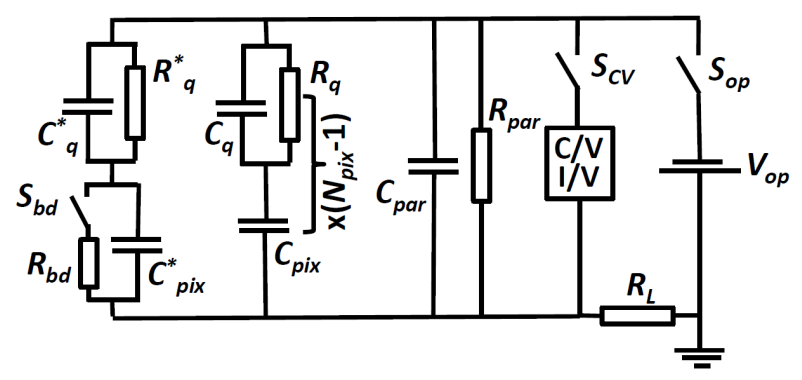

Fig. 4: $R C$ model of a reverse-biased SiPM. The symbols are explained in the text. To the left is the pixel with the Geiger discharge, in the middle the remaining $N_{p i x}-1$ pixels followed by a parasitic capacitance to account for the coupling of the biasing lines to the readout electrode of the SiPM, and a resistance for leakage currents outside of the pixels. $R_{L}$ is the load resistance representing the readout. The $C / V$ characteristics are simulated by connecting an $A C$-voltage via the switch $S_{C V}$ to the SiPM and the switches $S_{o p}$ and $S_{b d}$ open. The discharge of a pixel is simulated with switch $S_{C V}$ and $S_{o p}$ closed, by closing the switch $S_{b d}$ until the voltage over the pixel capacitance drops from the operating voltage $V_{o p}$ to the breakdown voltage $V_{b d}$ for $V_{o p}>V_{b d}$.

to the readout electrode of the SiPM, and the parasitic resistance, $R_{\text {par }}$, for the leakage current outside of the pixels. For the individual pixels $R_{q}$ denotes the quench resistor and $C_{q}$ the capacitive coupling between quench resistor and the square Al ring surrounding the pixel seen in Fig.2 The pixel capacitance is represented by $C_{p i x}$.

The Geiger discharge of the pixel denoted by "*" is described by the switch $S_{b d}$ which discharges $C_{p i x}$ via the resistor $R_{b d}$ until the voltage over the pixel, $V_{p i x}^{*}$, drops from the operating voltage $V_{o p}$ to the breakdown voltage $V_{b d} . R_{b d}$ is the effective resistance of the pixel discharge. The voltage transient over $R_{L}$ is obtained by solving the Kirchhoff equations with the switch $S_{C V}$ open and the switch $S_{o p}$ closed [23. The result is a system of three linear differential equations with the initial conditions: $V_{L}^{0}=0$, and $V_{p i x}^{* 0}=V_{p i x}^{0}=V_{o p}$, where $V_{L}$ is the voltage drop over $R_{L}$, and $V_{p i x}$ the voltage drop over the pixel capacitances $C_{p i x}$.

The main conclusions from the study of the analytic and numeric solutions are shortly summarized here. The time dependence of the output pulse, i.e. the current in the resistor $R_{L}$, has three parts: a fast rise until the Geiger discharge stops, a fast change to the third part, an exponential decrease until the voltage drop $V_{p i x}$ over the pixel reaches its initial value $V_{o p}$. For the numerical values given below, the following parameter values have been used: $V_{o p}=72.5 \mathrm{~V}, V_{b d}=69.5 \mathrm{~V}$, $N_{\text {pix }}=400, C_{\text {pix }}=90 \mathrm{fF}, R_{b d}=100 \mathrm{k} \Omega, R_{q}=125 \mathrm{k} \Omega$, and $R_{L}=50 \Omega$. For $C_{q}$, which is not well determined by our measurements, the results for two values, 0 and $10 \mathrm{fF}$, are given. These parameter values provide a fair description of the measurements presented in the following sections.

The main conclusions from the analysis of the model for the Geiger discharge are:

- During the Geiger discharge, which happens at the time scale of a fraction of a nanosecond, the voltage over the SiPM has to remain at the applied voltage $V_{o p}$. Otherwise a significant distortion of the measured pulse would occur, including situations with a significant dip in the pulse shape.

- The solutions of the equations are well behaved, meaning that there are no oscillatory solutions, and all exponents of the time dependencies have negative signs resulting in a stationary state for $t \rightarrow \infty$.

- Following Ref. [19] we assume that the Geiger discharge turns off when the discharge current drops below the turn-off current $I_{o f f}$, where typical values are between 0.1 and $1 \mathrm{~mA}$. This allows us to estimate the value of $R_{b d} \approx V_{b d} / I_{o f f}$.

- For the switch-off time of the Geiger discharge, $t_{\text {off }}$, we find under the assumption $R_{L} \ll R_{b d}$ 


$$
t_{o f f}=R_{b d} \cdot\left(C_{p i x}+C_{q}\right) \cdot \ln \left(V_{o p} / V_{b d}\right) \quad(380,423 \mathrm{ps}) .
$$

Here and below the numerical values for the parameter values discussed above are given in parentheses for $C_{q}=0$ and $10 \mathrm{fF}$.

- For the decay time $\tau_{\text {slow }}$ of the signal we find

$$
\tau_{\text {slow }}=\frac{R_{q}\left(C_{p i x}+C_{q}\right)+N_{p i x} R_{L} C_{p i x}}{1+\frac{N_{p i x} C_{p i x} C_{q} R_{q} R_{L}}{\left(\left(C_{p i x}+C_{q}\right) R_{q}+N_{p i x} C_{p i x} R_{L}\right)^{2}}} \quad(13.05,14.14 \mathrm{~ns}) .
$$

For the values of the parameters used the correction term in the denominator for $C_{q}=10 \mathrm{fF}$ is $0.6 \%$.

- For the integral of the current flowing through $R_{L}, Q_{t o t}$, we could not find a general expression. In the parameter domain relevant for the SiPMs studied here, the following parametrization describes the numerical results to an accuracy of better than $1 \%$.

$$
Q_{t o t}=\left(C_{p i x}+C_{q}\right)\left(V_{o p}-V_{b d}\right)\left(1+0.02 \cdot \frac{R_{b d}}{R_{q}}\right) \quad(274,305 \mathrm{fC})
$$

- For the fast component of the signal, $Q_{\text {fast }}$, defined as the integrated excess of the signal above the exponential with decay time $\tau_{\text {slow }}$, we again could not find a general expression. The following parametrization describes the numerical results with an accuracy of a few fC in the relevant parameter domain:

$$
Q_{f a s t}=2 / 3 \cdot C_{q}\left(V_{o p}-V_{b d}\right) \quad(0,20 \mathrm{fC}) .
$$

We note here that for $C_{q}=0 \mathrm{fF}$, the numerical model calculation gives a small negative value of $-4 \mathrm{fC}$, and certainly more work is needed to find a satisfactory parametrization for $Q_{\text {fast }}$.

For voltages below $V_{b d}$ the switches $S_{b d}$ and $S_{o p}$ are open, and $S_{C V}$, which connects the SiPM either to a voltage-source/current-meter or to a capacitor bridge, closed, and the complex resistance of a SiPM with $N_{p i x}$ pixels below the breakdown voltage is given by:

$$
\left(\frac{1}{R_{p a r}}+i \omega C_{p a r}+N_{p i x} \cdot\left(\frac{1}{i \omega C_{p i x}}+\frac{R_{q}}{1+i \omega C_{q} R_{q}}\right)^{-1}\right)^{-1}
$$

In this work we determine the parameters of the equivalent circuit in different ways, in order to check the validity of the model and obtain reliable values of the relevant parameters for both irradiated and non-irradiated SiPMs.

\subsection{Forward current}

Fig. 5a shows the forward current of the SiPMs at room temperature before irradiation, and after irradiation to $200 \mathrm{~Gy}, 2 \mathrm{MGy}$, and $20 \mathrm{MGy}$. For forward voltages below $750 \mathrm{mV}$, a significant increase in current as function of dose is observed. For a diode in series with a resistor $R_{s}$ we expect the following dependence of the forward current, $I$, on the forward voltage $V$ 25]

$$
I=I_{d 0}\left(e^{\frac{q_{0} V_{d}}{k_{B} T}}-1\right)+I_{r 0}\left(e^{\frac{q_{0} V_{d}}{2 \cdot k_{B} T}}-1\right), V=V_{d}+I \cdot R_{s},
$$

where the voltage drop over the diode is denoted by $V_{d}$. The first term describes the diffusion current with the parameter $I_{d 0}$, and the second term the recombination current with the parameter $I_{r 0}$. The elementary charge is denoted by $q_{0}$, and the term $I \cdot R_{s}$ is the voltage drop over the series resistor $R_{s}$. The absolute temperature is $T$, and $k_{B}$ the Boltzmann constant. 
Table 1: Results of the fit to the forward current for the non-irradiated SiPM. The value of the quench resistor of the $\mathrm{SiPM}$ model is obtained from $R_{q}^{\text {forw }}=N_{\text {pix }} \cdot R_{s}$. The first error gives the uncertainty estimated for an individual SiPM, the second the spread between the SiPMs.

\begin{tabular}{ccccc}
\hline$I_{d 0}[\mathrm{fA}]$ & $I_{r 0}[\mathrm{pA}]$ & $T[\mathrm{~K}]$ & $R_{s}[\Omega]$ & $R_{q}^{\text {forw }}[\mathrm{k} \Omega]$ \\
\hline $6.0 \pm 0.9 \pm 2.0$ & $1.4 \pm 0.1 \pm 0.3$ & $300.6 \pm 0.5 \pm 0.5$ & $353 \pm 5 \pm 10$ & $141 \pm 2 \pm 4$ \\
\hline
\end{tabular}

The solid line in Fig.5 a shows a fit of Eq.66 to the forward current of the non-irradiated SiPM. The data are well described over nine orders of magnitude. For the parameters the values shown in Table1 are obtained1

We note that even at the forward voltage of $2 \mathrm{~V}$ the measured differential resistance $(\mathrm{d} I / \mathrm{d} V)^{-1}$ is as high as $550 \Omega$, implying a significant extrapolation to obtain the value of $R_{s}$ and a systematic uncertainty which is difficult to estimate. Assuming that the entire forward current flows through the $N_{p i x}=400$ pixels of the SiPM, a value for the quenching resistance of $R_{q}^{\text {forw }}=141 \pm 2 \pm 4 \mathrm{k} \Omega$ is obtained. The superscript marks the method used to determine $R_{q}$.

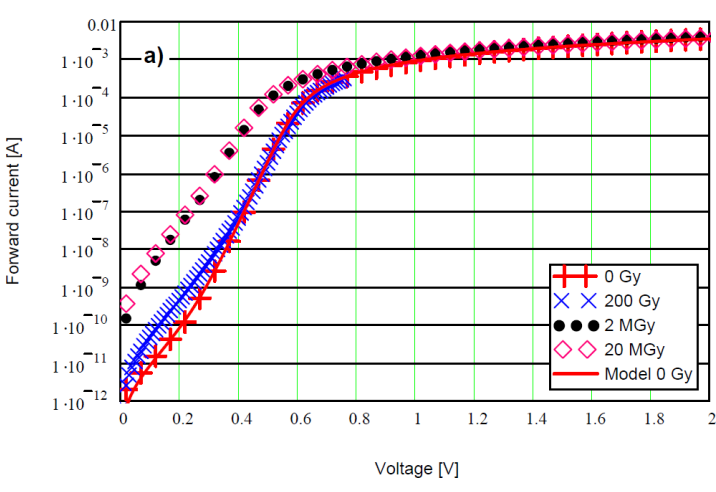

(a)

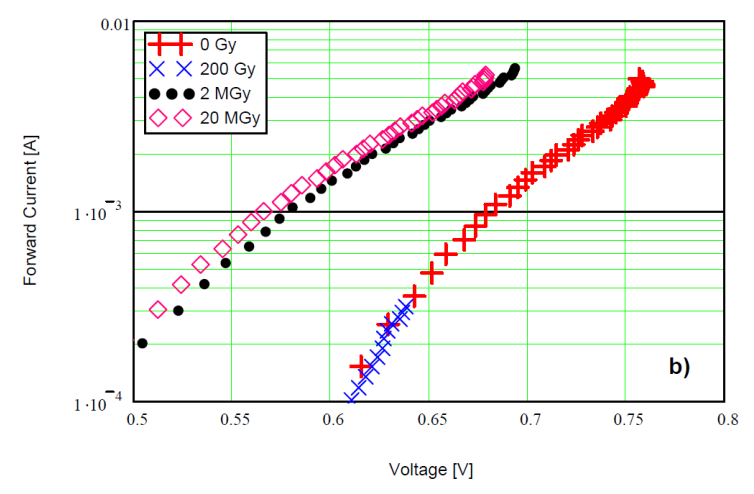

(b)

Fig. 5: Forward current for different X-ray doses (a) as function of the applied voltage (the solid line is the fit of the 0 Gy data using Eq. 6), (b) as function of the applied voltage minus the voltage drop over the series resistance $R_{s}$ calculated according to the second part of Eq.6.

We note that for the higher voltage values the slopes of the $I-V$ curves for the non-irradiated and the irradiated sensor are similar. We conclude, that the value of $R_{q}$ does not change significantly with X-ray irradiation.

Fig. 5b shows the forward current as function of $V-I \cdot R_{s}$, the expected voltage drop over the diode. The observed current increase, e.g. $2.5 \mathrm{~mA}$ at $0.65 \mathrm{~V}$ between $0 \mathrm{~Gy}$ and $2 \mathrm{MGy}$, is orders of magnitude larger than the estimated upper limit of $25 \mathrm{nA}$ for the surface generation current given in Section 3.1. We explain the apparent increase in current by a decrease of the potential of the $p$-epi layer due to radiation-induced positive oxide charges, which causes the voltage drop over the buried $n^{+} p$ junction to increase by about $0.1 \mathrm{~V}$. As radiation-induced positive oxide charges, which typically reach densities of several $10^{12} \mathrm{~cm}^{-2}$ for doses above $100 \mathrm{kGy}$, shift the flat-band voltage to negative values, well beyond the applied forward voltage, we expect that an electron-accumulation layer forms at the $\mathrm{Si}-\mathrm{SiO}_{2}$ interface. Thus we expect hardly any surface generation current, and the apparent increase in forward current is due to a change of the voltage drop over the buried

\footnotetext{
${ }^{1}$ The values have been obtained by minimizing the sum of the squares of the differences between the model calculation and the measured values divided by the measured values. As these differences are dominated by systematic uncertainties, like a required accuracy of about $1 \mathrm{mV}$ for the voltage steps, only a crude error estimation is possible. The errors have been estimated by varying the voltage range of the fit.
} 
Table 2: Reverse current, $I_{r e v}$, and current divided by the $\mathrm{Si}_{-} \mathrm{SiO}_{2}$-interface area, $J_{\text {rev }}$, at $40 \mathrm{~V}$ as function of X-ray dose. As the currents for the two SiPMs irradiated to 20 MGy are very different, both values are shown.

\begin{tabular}{ccccccc}
\hline Dose & 0 Gy & 200 Gy & 20 kGy & 2 MGy & 20 MGy & 20 MGy \\
\hline$I_{\text {rev }}[\mathrm{pA}]$ & 10 & 400 & 5800 & 15000 & 12000 & 40000 \\
$J_{\text {rev }}\left[\mathrm{nA} / \mathrm{cm}^{2}\right]$ & 1.5 & 60 & 1000 & 2500 & 2000 & 6500 \\
\hline
\end{tabular}

$n^{+} p$ junction for a given applied voltage. We also have observed that for the irradiated SiPMs the measured forward current depends on the ramping speed of the voltage. We interpret this as the result of radiation-induced border traps [24, and possiblyz some heating.

\subsection{Reverse current}

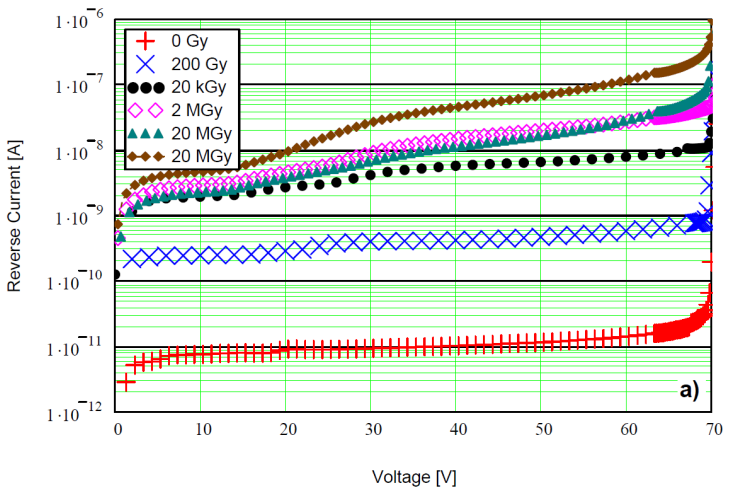

(a)

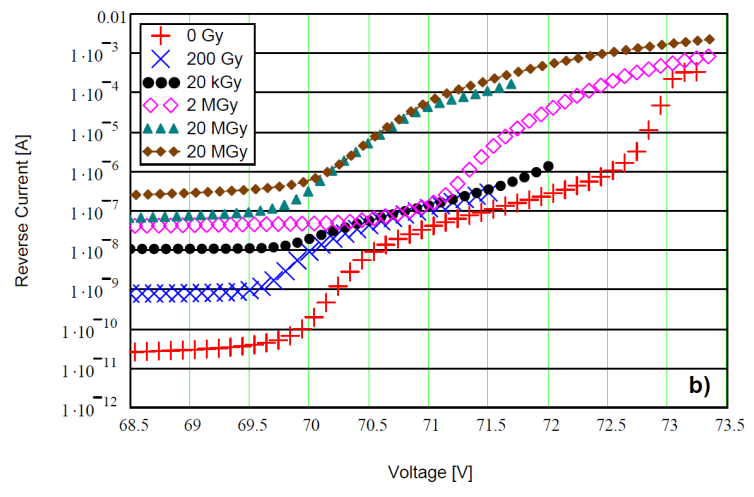

(b)

Fig. 6: Reverse currents of SiPMs as function of voltage before, and after irradiation with X-rays to $200 \mathrm{~Gy} 20 \mathrm{kGy}, 2 \mathrm{MGy}$, and $20 \mathrm{MGy}$ a) below the breakdown voltage, and b) in the region of and above the breakdown voltage.

Fig.6 6 shows the reverse currents, $I_{\text {rev }}$, for several SiPMs before irradiation, and after irradiation to $200 \mathrm{~Gy}, 20 \mathrm{kGy}, 2 \mathrm{MGy}$, and $20 \mathrm{MGy}$. As shown in Fig.6]a, $I_{\text {rev }}$ increases by several orders of magnitude with X-ray dose below the breakdown voltage, $V_{b d}$, which is about $69.5 \mathrm{~V}$ (see Section 3.7). For a voltage of $40 \mathrm{~V}$, which is well above the depletion voltage of approximately $22 \mathrm{~V}$, the values of $I_{r e v}$ and of $J_{r e v}$, the current divided by the $\mathrm{Si}_{-} \mathrm{SiO}_{2}$ area, are given in Table 2 . For unknown reasons, there is a difference by a factor three for the two SiPMs irradiated to 20 MGy. We therefore show the results from both samples. The values roughly agree with the expectations from surface currents, if we assume that most of the $\mathrm{Si}_{-} \mathrm{SiO}_{2}$ area is depleted. We conclude that the reverse current below $V_{b d}$ is dominated by the surface-generation current from the $\mathrm{Si}_{-} \mathrm{SiO}_{2}$ interface, which increases with X-ray dose until it saturates at higher dose values [13 15].

Above $V_{b d}$, where the SiPMs are operated, the situation is more complex. The results are shown in Fig. 6b. The curves for $200 \mathrm{~Gy}$ and $20 \mathrm{kGy}$ can be well (within $20 \%$ ) described by the sum of the 0 Gy curve plus the current at $60 \mathrm{~V}$. The behavior of the $2 \mathrm{MGy}$ data is similar up to about $71 \mathrm{~V}$. At higher voltages however, the current increases rapidly, then flattens, and finally at $73 \mathrm{~V}$ reaches a value which is approximately a factor two above the value for the non-irradiated SiPM. Finally, starting around $70 \mathrm{~V}$ the SiPMs irradiated to $20 \mathrm{MGy}$ show a steady increase of the reverse current which reaches a value about a factor four above the value of the non-irradiated SiPM at $73 \mathrm{~V}$. We note that the two SiPMs irradiated to $20 \mathrm{MGy}$ show similar currents above $V_{b d}$. We conclude that above $V_{b d}$ the dose dependence is fairly complicated. In particular for irradiations of 
2 and 20 MGy there is clear evidence, that part of the surface current is amplified. We will come back to this topic when we discuss the measurements of the dark-count rate in Section 3.8.

Fig. 7 shows for the different SiPM samples investigated the reverse current, $I_{\text {rev }}$, below the breakdown voltage before and after X-ray irradiation. We notice a number of steps in the $I-V$ characteristics: A first step around $17 \mathrm{~V}$, which appears to be approximately independent of X-ray dose, and a second step, which is not visible at $0 \mathrm{~Gy}$, is at $22 \mathrm{~V}$ for a dose of $200 \mathrm{~Gy}$, and at $26 \mathrm{~V}$ for dose values of $20 \mathrm{kGy}$ and above. We speculate that the first step is due a "sudden" increase of the depletion volume of the bulk below the $\mathrm{Si}_{-} \mathrm{SiO}_{2}$ interface, which results in an increase of the bulk-generation current, and that the second step is due to an increase of the depleted $\mathrm{Si}-\mathrm{SiO}_{2}$-interface, resulting in an increase in surface-generation current. As we do not have the necessary information of the SiPM design, we are not able to check these highly speculative guesses with TCAD simulations.

We attribute the increase in current for reverse voltages between the depletion voltage and the breakdown voltage to the onset of charge multiplication of the generation currents. It has been checked, that the increase in current is compatible with the ionization integrals for electrons calculated with the electric field estimated from the capacitance/voltage measurements presented in Section 3.6

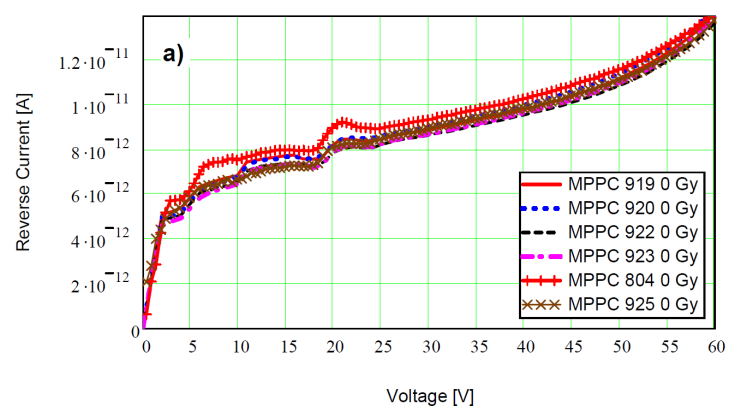

(a)

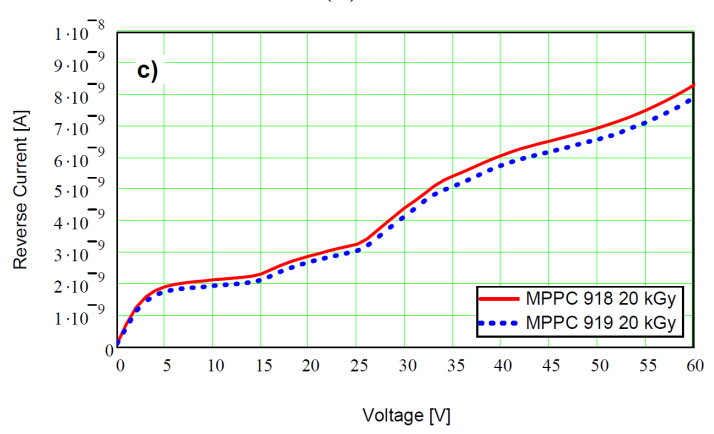

(c)

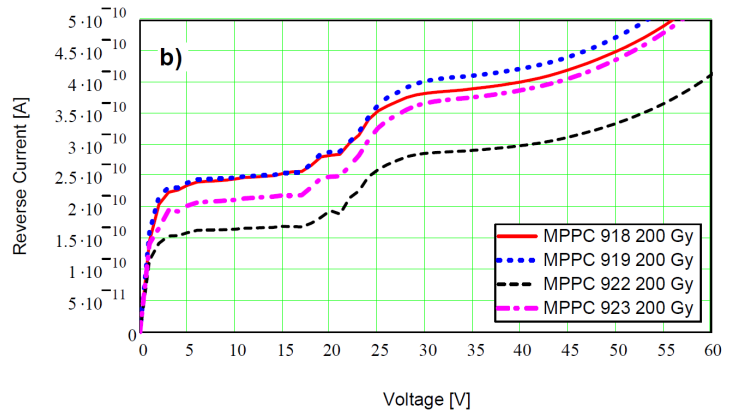

(b)

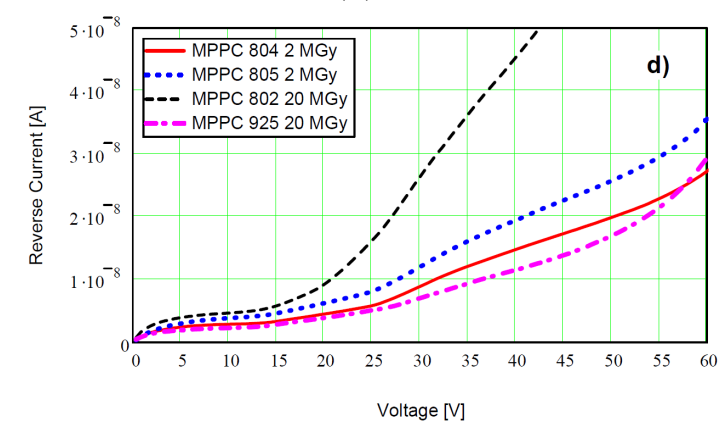

(d)

Fig. 7: Reverse current below the breakdown voltage for the different SiPM samples (a) before irradiation, (b) after irradiation to $200 \mathrm{~Gy}$, (c) to $20 \mathrm{kGy}$, and (d) to 2 and $20 \mathrm{MGy}$.

\subsection{Capacitance and conductance versus frequency}

The measurement of the capacitance and conductance as function of frequency allows determining parameters of the equivalent circuit presented in Section 3.2, and studying their dependencies on voltage and $\mathrm{X}$-ray dose. Charge traps at the $\mathrm{Si}_{-} \mathrm{SiO}_{2}$ interface, which are not included in the equivalent circuit, can also result in changes of the frequency dependence of capacitance and conductance. We first present the analysis method and results for the non-irradiated SiPMs, and then the dependence of the parameters of the equivalent model on X-ray dose. The measurements 
Table 3: Dose dependence of SiPM parameters determined from the capacitance-frequency measurements at $67 \mathrm{~V}$.

\begin{tabular}{cccccc}
\hline Dose & 0 Gy & 200 Gy & 20 kGy & 2 MGy & 20 MGy \\
\hline$R_{\text {par }}[\mathrm{M} \Omega]$ & $2100 \pm 100$ & $2000 \pm 100$ & $1600 \pm 80$ & $275 \pm 50$ & $75 \pm 20$ \\
$R_{q}^{C f}[\mathrm{k} \Omega]$ & $125 \pm 5$ & $116 \pm 5$ & $112 \pm 5$ & $110 \pm 5$ & $108 \pm 5$ \\
$C_{\text {pix }}^{C f}[\mathrm{fF}]$ & $94.0 \pm 1.5$ & $93.8 \pm 1.5$ & $93.5 \pm 1.5$ & $93.0 \pm 1.5$ & $93.5 \pm 1.5$ \\
$R_{q}^{C f} \cdot C_{\text {pix }}^{C f}[\mathrm{~ns}]$ & $11.8 \pm 0.6$ & $10.9 \pm 0.6$ & $10.5 \pm 0.6$ & $10.2 \pm 0.6$ & $10.1 \pm 0.6$ \\
\hline
\end{tabular}

have been performed for frequencies between $100 \mathrm{~Hz}$ and $2 \mathrm{MHz}$ and reverse voltages between $2 \mathrm{~V}$ and $67 \mathrm{~V}$. A HP $4263 \mathrm{LCR}$ meter has been used. The amplitude of the AC voltage has been $500 \mathrm{mV}$.

Fig.8 shows for a non-irradiated SiPM for the three voltages 2,7 , and $67 \mathrm{~V}$ the results of the measurement of the complex resistance. The LCR meter gives us the parallel resistance, $R_{p}$, and the parallel capacitance, $C_{p}$, as function of frequency $f$. In addition to $R_{p}(f)$ and $C_{p}(f)$ we also show the series resistance $R_{\text {ser }}(f)$ and the series capacitance $C_{\text {ser }}(f)$, obtained from $Z=R_{\text {ser }}+1 /\left(i \cdot 2 \pi f \cdot C_{\text {ser }}\right)=\left(1 / R_{p}+i \cdot 2 \pi \cdot f \cdot C_{p}\right)^{-1}$. With the help of Eq. 5 direct insight into the values of the elements of the equivalent circuit shown in Fig. 4 can be gained. For the initial discussion we assume that the values of the parasitic capacitance, $C_{p a r}$, and of the quench capacitance, $C_{q}$, are sufficiently small and can be ignored.

Fig.s $8 \mathrm{a}$ and $\mathrm{b}$ show that, for a given voltage, below $1 \mathrm{MHz}$ the values of the series and parallel capacitances are the same and independent of the frequency. They decrease with increasing voltage, as expected from the increase in depletion depth. Above the depletion voltage, which is about $22 \mathrm{~V}$, the rate of decrease slows down, and the results at $67 \mathrm{~V}$ are representative for the data between the depletion and the breakdown voltage. The measured value of $37.6 \pm 0.6 \mathrm{pF}$ corresponds to a single-pixel capacitance $C_{\text {pix }}^{C f}=94.0 \pm 1.5 \mathrm{fF}$, where the superscript indicates the method used to determine the pixel capacitance.

Fig. $8 \mathrm{c}$ shows that the series resistance, $R_{\text {ser }}$, decreases up to frequencies of about $10 \mathrm{kHz}$, where it becomes independent of frequency and voltage. This can be understood from Fig.4 at high frequencies $R_{p a r}$ can be ignored and only the pixel capacitance, $C_{p i x}$, in series with the quench resistance, $R_{q}$, is relevant. The value found at $67 \mathrm{~V}$ is $R_{\text {ser }}=310 \pm 15 \Omega$, which corresponds to $R_{q}^{C f}=125 \pm 5 \mathrm{k} \Omega$, which is close to the value of $141 \pm 6 \mathrm{k} \Omega$ from the forward current measurements reported in Section 3.3 .

Fig. $8 \mathrm{~d}$ shows that for frequencies below $\approx 2 \mathrm{kHz}$ the measured parallel resistance is essentially independent of frequency. This is expected from the $R C$ model described by Eq.5, as at low frequencies the circuit corresponds to the parasitic resistance, $R_{p a r}$, in parallel with the pixels. The values found for $R_{\text {par }}$ are $0.79,1.1$, and $2.2 \mathrm{G} \Omega$ for voltages of 7,12 , and $67 \mathrm{~V}$, with an estimated uncertainty of about $10 \%$.

The curves shown in figure 8 are obtained from the model using the measured values of the parallel resistance at $800 \mathrm{~Hz}$ for $R_{\text {par }}, N_{\text {pix }} \cdot R_{\text {ser }}$ at $100 \mathrm{kHz}$ for $R_{q}, C_{\text {ser }} / N_{\text {pix }}$ at $10 \mathrm{kHz}$ for $C_{\text {pix }}$, and $N_{p i x}=400$ for the number of pixels. The values and the errors estimated from the spread of the measurements in the relevant frequency range, are reported in Table3. The model provides a fair overall description of the measurements for most of the frequency range. Larger deviations are found for frequencies below $200 \mathrm{~Hz}$ and at $2 \mathrm{MHz}$, where the precise measurement of the complex resistance is difficult.

Next the sensitivity of the data to the circuit elements $C_{q}$ and $C_{p a r}$, which have been ignored in the discussion above, is investigated. They are varied in the model calculations and the results compared to the data by inspecting the histograms and the mean relative deviations to the model. We find $C_{q}<0.5 \mathrm{pF}$, and $C_{\text {par }}<1.5 \mathrm{nF}$. These limits are not very stringent and thus of limited significance.

A similar analysis as presented above has also been performed for the SiPMs after X-ray 


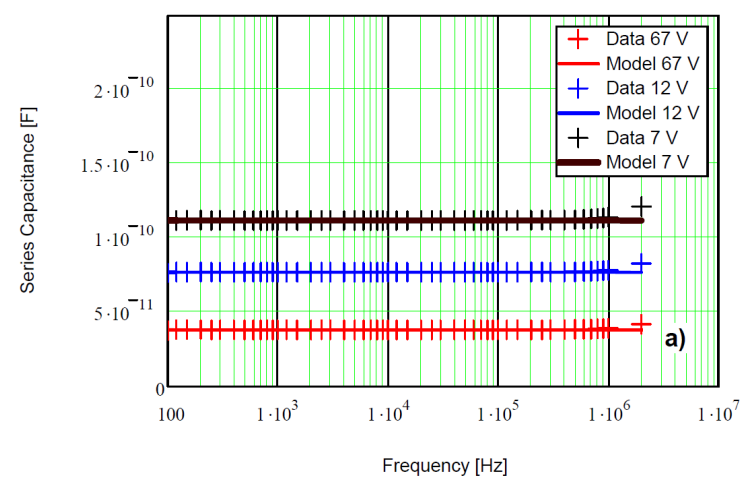

(a)

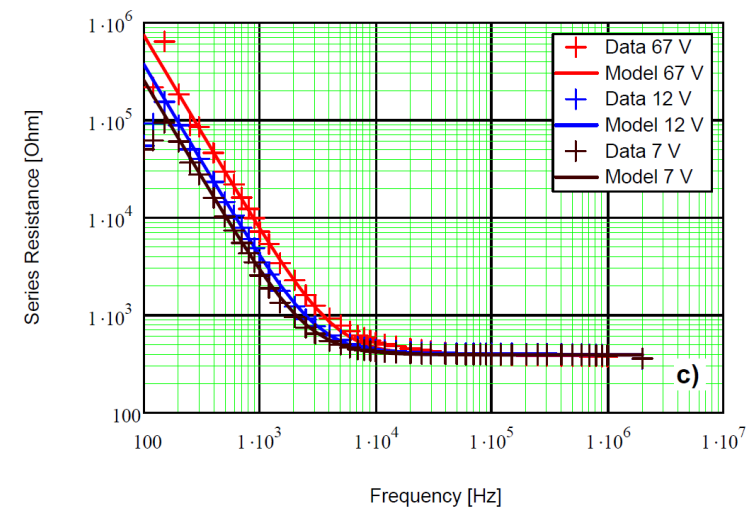

(c)

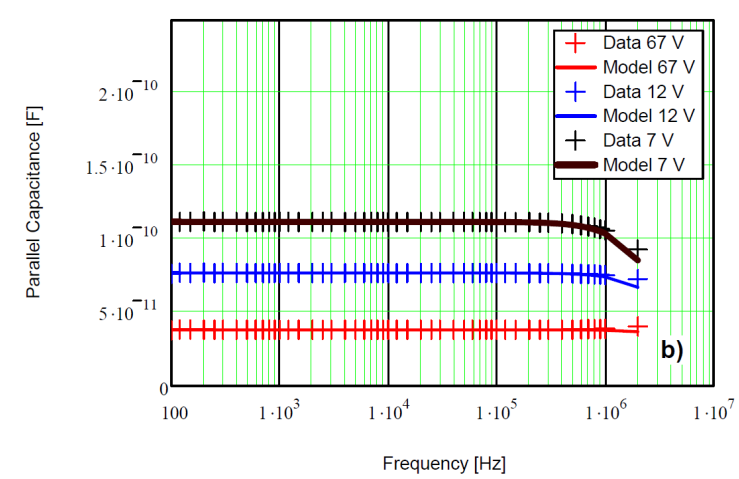

(b)

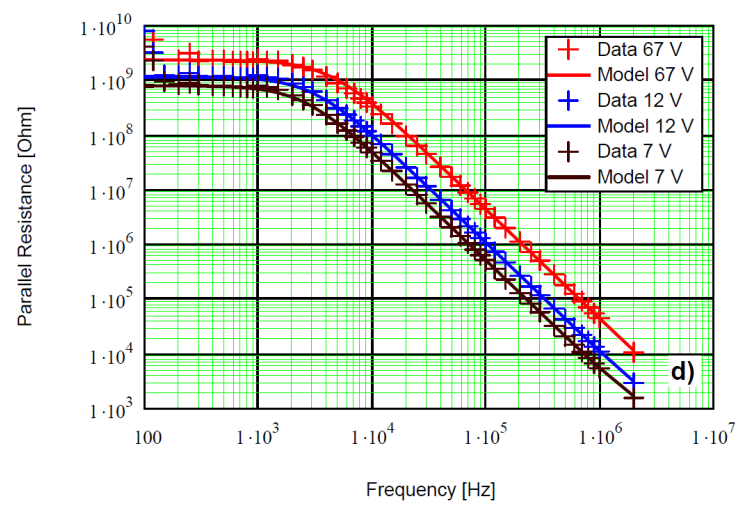

(d)

Fig. 8: Capacitance and resistance as function of frequency for the same SiPM before X-ray irradiation at 7,12 , and $67 \mathrm{~V}$. The data are shown as crosses and the model calculations as lines. (a) Series capacitance, (b) parallel capacitance, (c) series resistance, and (d) parallel resistance.

irradiation to $200 \mathrm{~Gy}, 20 \mathrm{kGy}, 2 \mathrm{MGy}$, and $20 \mathrm{MGy}$. Table 3 shows as function of dose the values obtained from the $C f$ measurements at $67 \mathrm{~V}$ for the parasitic resistance $R_{\text {par }}$, the quenching resistor $R_{q}^{C f}$, and the pixel capacitance $C_{\text {pix }}^{C f}$. The time constant $R_{q}^{C f} \cdot C_{\text {pix }}^{C f}$ is approximately the recharging time of a single discharged pixel ignoring the effects of the load resistance $R_{L}$ and the additional $N_{\text {pix }}-1$ pixels.

We note that the value of $R_{\text {par }}$ decreases steadily with increasing X-ray dose, and that the value at $20 \mathrm{MGy}$ is about a factor 30 smaller than for $0 \mathrm{~Gy}$. The values of $R_{\text {par }}$ are several orders of magnitude smaller than what would be expected from the reverse current: at 0 Gy the slope of the reverse current $\mathrm{d} I_{\text {rev }} / \mathrm{d} V=0.5 \mathrm{pA} / \mathrm{V}$ at $67 \mathrm{~V}$, which corresponds to a resistance of $2 \mathrm{~T} \Omega$, whereas the value from the $C f$ measurement is $2 \mathrm{G} \Omega$.

Within the measurement accuracy of about $1.5 \%$, the value of $C_{\text {pix }}^{C f}$ is independent of dose. The data however, indicate a decrease at the percent level between $0 \mathrm{~Gy}$ and the highest dose values. The values found for $R_{q}^{C f}$ also decrease with dose: the value at 20 MGy is about $15 \%$ smaller than the value at $0 \mathrm{~Gy}$.

Next we present results for the series capacitance, $C_{s e r}$, and the dissipation, $D$, for reverse voltages between 0 and $30 \mathrm{~V}$, where the SiPM gets fully depleted and structures in the reverse current, as discussed in Section 3.4, are observed. The dissipation $D=2 \pi f \cdot C_{s e r} \cdot R_{s e r}$ is a measure for the power dissipation in a $R C$ circuit with series capacitance $C_{\text {ser }}$ and series resistance $R_{\text {ser }}$.

Fig. 9 shows the results at $10 \mathrm{kHz}$. For reverse voltages below $8 \mathrm{~V}$ and above $20 \mathrm{~V}$, the capacitance values are approximately independent of dose. Between $8 \mathrm{~V}$ and $20 \mathrm{~V}$ they decrease with X-ray dose. We explain this by radiation-induced positive oxide charges, which change the potential at the 


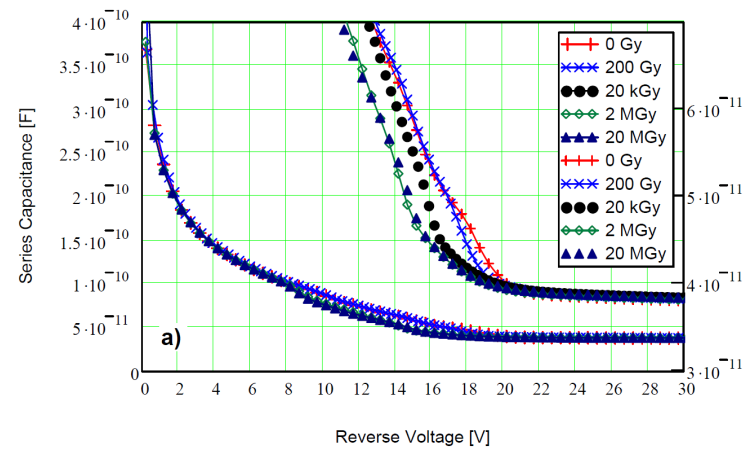

(a)

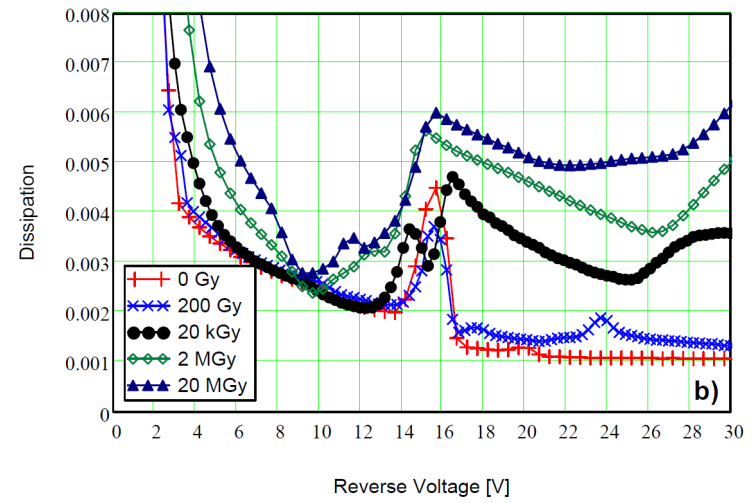

(b)

Fig. 9: Capacitance a) and dissipation b) measured at $10 \mathrm{kHz}$ versus voltage for the same SiPM before and after irradiation to $200 \mathrm{~Gy}, 20 \mathrm{kGy}, 2 \mathrm{MGy}$, and $20 \mathrm{MGy}$. For better visibility the capacitance values in a) are shown twice, with an expanded scale and suppressed zero on the right.

$\mathrm{Si}-\mathrm{SiO}_{2}$ interface in a way that full depletion is reached at a lower voltage. The radiation-induced oxide-charge density is known to saturate at dose values between $100 \mathrm{kGy}$ and $1 \mathrm{MGy}$ 13, which explains why we observe the same capacitance values at $2 \mathrm{MGy}$ and at $20 \mathrm{MGy}$.

The dissipation, D, shown in Fig.9 b, shows complex structures with strong dependencies on $\mathrm{X}$-ray dose. They appear in the same voltage range where structures in the current shown in Fig. 7 are observed. We therefore assume that they are related to changes of the depletion zone close to the $\mathrm{Si}-\mathrm{SiO}_{2}$ interface, to changes in the charge layers at the $\mathrm{Si}-\mathrm{SiO}_{2}$ interface, and above the depletion voltage at higher doses, to charge transfer to the $\mathrm{Si}_{-} \mathrm{SiO}_{2}$-interface states. However, we lack a real understanding of these observations.

\subsection{Capacitance versus voltage and doping profile}

In the one-sided abrupt junction approximation [25], for the structure shown in Fig. 3, the doping density $N_{e f f}$ in the $p$-doped region is approximately equal to the majority charge carrier density, $p$, which can be obtained from the capacitance, $C$, and the effective pixel area, $A_{e f f}$ :

$$
N_{e f f}(x) \approx p(V(x))=\frac{2}{q_{0} \cdot \epsilon_{S i} \cdot\left(A_{e f f}(V)\right)^{2}} \cdot\left(\frac{\mathrm{d}\left(1 / C(V)^{2}\right)}{\mathrm{d} V}\right)^{-1}
$$

where the distance from the $n^{+} p$ junction, $x$, is related to the voltage $V$ by:

$$
x(V)=\frac{\epsilon_{S i} \cdot A_{e f f}(V)}{C(V)}=\frac{\epsilon_{S i} \cdot N_{p i x} \cdot A_{p i x} \cdot \operatorname{corr}(V)}{C(V)} .
$$

The dielectric constant of silicon is $\epsilon_{S i}$. A voltage dependent $A_{e f f}$ is introduced, to account for the change of the geometry of the depleted region with voltage. The effective doping density, $N_{\text {eff }}$, is approximately equal to the majority carrier density, $p(x)$, if changes in doping density occur more gradually than the extrinsic Debye length $L_{D}[26$. We will come back later to this point.

Fig. 10 a shows $1 / C^{2}$ at $10 \mathrm{kHz}$ for the SiPM before irradiation, and after irradiation to $200 \mathrm{~Gy}$, $20 \mathrm{kGy}, 2 \mathrm{MGy}$, and $20 \mathrm{MGy}$. For a diode with uniform doping and constant area, the dependence of $1 / C^{2}$ on voltage is linear until the depletion voltage is reached. The observed dependence however is very different. As the $1 / C^{2}$ curves for the different dose values are similar, only the results of the analysis of the $0 \mathrm{~Gy}$ data are shown in the following.

Next we present our model for $A_{e f f}(V)$, the voltage-dependent effective area of the SiPM capacitance which is used to extract the doping profile from the capacitance measurement. Assuming 


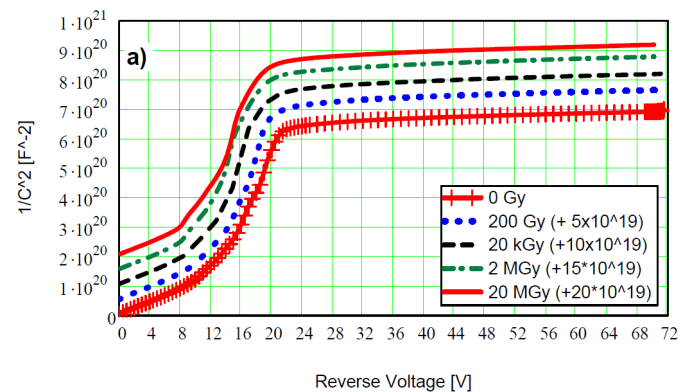

(a)

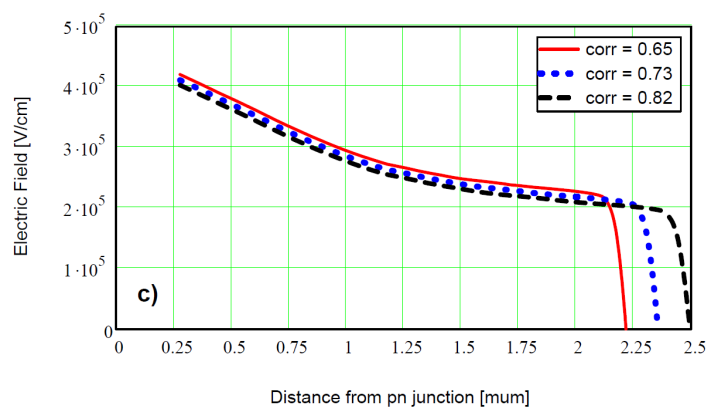

(c)

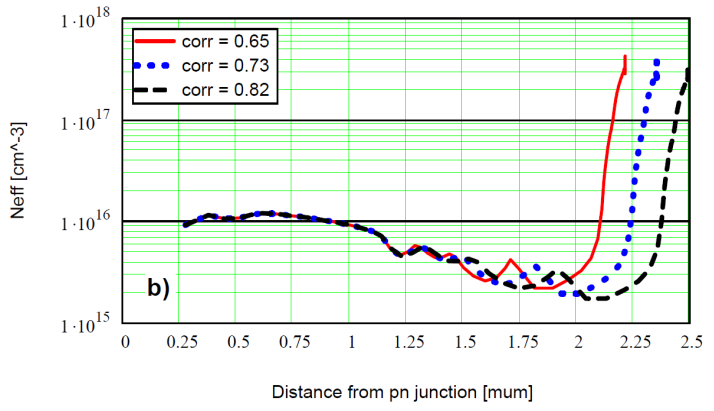

(b)

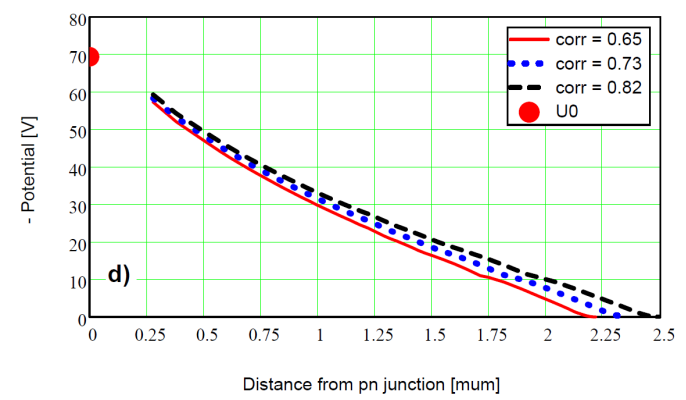

(d)

Fig. 10: (a) $1 / C^{2}$ at $10 \mathrm{kHz}$ versus voltage before irradiation, and after irradiation to $200 \mathrm{~Gy}, 20 \mathrm{kGy}$, $2 \mathrm{MGy}$, and $20 \mathrm{MGy}$. For better visibility the curves are shifted vertically in steps of $5 \cdot 10^{19} \mathrm{~F}^{-2}$. (b) Effective dopant density, (c) electric field, and (d) potential as function of the distance from the $p n$ junction reconstructed from the 0 Gy data for different correction factors for the effective pixel capacitance.

the SiPM structure shown in Fig. 3, the pn junction is buried some distance away from the SiPM surface. Thus at low voltages, practically the entire region below the pixels will be non-depleted, and $A_{\text {eff }}$ is given by the SiPM area $N_{p i x} \cdot A_{p i x}$ with the pixel area $A_{p i x}=(50 \mu \mathrm{m})^{2}$. Once the SiPM is fully depleted, the situation is different: 400 disconnected pixels, each with the area of the $p^{+}$implant. We denote the effective area of the corresponding capacitance corr $\cdot N_{p i x} \cdot A_{p i x}$.

To estimate corr , the ratio of the capacitor with the $p^{+}$-implant area to the capacitor with area $A_{p i x}$ and no edge corrections, the edge correction for a circular plate capacitor derived by Kirchhoff in Ref. 27] has been used. We thus assume that the edge correction of a square capacitor and of a circular capacitor is the same if they have the same circumference. The capacitance between the $p^{+}$-pixel implant and the non-depleted $n^{+}$substrate at distance $d$ (see Fig. 33 is calculated as twice the capacitance including edge effects of a capacitor with the area of the $p^{+}$implant and plate distance $2 d$. Assuming an area of $A_{p i x}=(50 \mu \mathrm{m})^{2}$ for the pixel, and an area of $(40 \mu \mathrm{m})^{2}$ for the $p^{+}$ implant, we obtain a value of corr $=0.73$ for a depletion depth of $d=2 \mu \mathrm{m}$. The values for pixels of area $(37.5 \mu \mathrm{m})^{2}$ and $(42.5 \mu \mathrm{m})^{2}$ are 0.65 and 0.82 , respectively. For $A_{e f f}(V)$ we have assumed $N_{p i x} \cdot A_{p i x}$ for voltages below $V_{0}=10 \mathrm{~V}, N_{p i x} \cdot A_{p i x} \cdot \operatorname{corr}$ above $V_{1}=18 \mathrm{~V}$, and a linear voltage dependence in between. We find, that the results on the effective doping depend only weakly on the choice of $V_{0}$ and $V_{1}$.

Fig. 10 b shows for 3 values of corr the dependence of the effective doping $N_{\text {eff }}$ on the distance $x$ from the buried $p n$ junction derived using Eqs.77 and8. The wiggles of $N_{\text {eff }}$ show that the assumption for the voltage dependence of $A_{\text {eff }}$ is only an approximation. It can be seen from Eq.8 that an increase in corr results in a proportional increase of the value obtained for the full depletion depth. The full depletion is reached at about $2.3 \mu \mathrm{m}$, and the doping in the $p$-epitaxial layer (see Fig. 3 is between $3 \cdot 10^{15}$ and $10^{16} \mathrm{~cm}^{-3}$. For the $p^{+}$region values up to 3 and $5 \cdot 10^{17} \mathrm{~cm}^{-3}$ are 


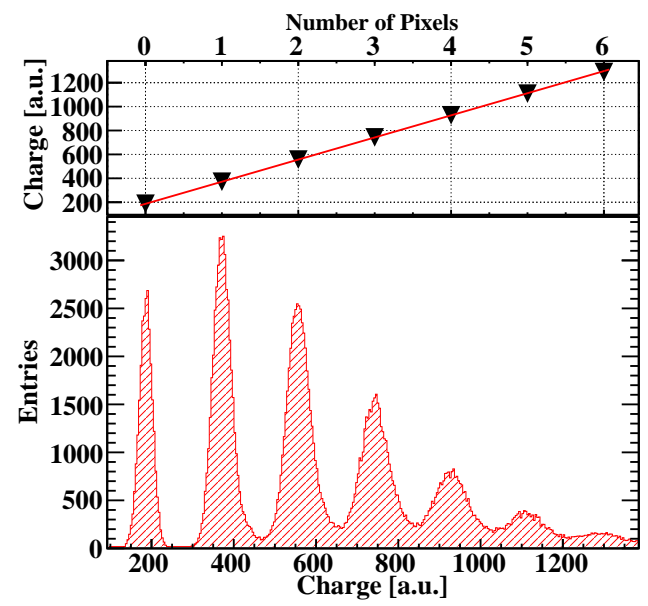

Fig. 11: Determination of the gain and breakdown voltage for the SiPM MPPC 919. Shown are the pulse-area spectrum in QDC units for the non-irradiated SiPM measured at $71.5 \mathrm{~V}$ with a fit to the spectrum for the determination of the relation between the number of discharging pixels, $n_{\text {pix }}$, and pulse area.

found. As the extrinsic Debye length for a doping of $2 \cdot 10^{17} \mathrm{~cm}^{-3}$ of $L_{D} \approx 0.01 \mu \mathrm{m}$ is similar to the observed width of the doping step, correcting for the diffusion of the majority charge carriers will increase the sharpness of the step and the maximum value determined for $N_{e f f}$. Integration of $N_{\text {eff }}$ yields the electric field $E$, and integration of $E$ the negative potential, as shown in Fig.s 10 c and $\mathrm{d}$. In addition, the voltage $U_{0}=69.4 \mathrm{~V}$, up to which the analysis has been made, is shown as dot at the position of the $p n$ junction.

The values and position dependencies found for the doping and the electric field appear realistic. However, a detailed knowledge of doping profiles and of the SiPM design would be required to check the validity of the analysis presented.

\subsection{Gain and breakdown voltage}

For the measurement of the gain, $G$, and of the breakdown voltage, $V_{b d}$, the method described in Ref. 28] has been used. The SiPMs have been illuminated by a pulsed LED with a wavelength of $405 \mathrm{~nm}$ and a pulse width at FWHM of about $1 \mathrm{~ns}$. The light intensity was chosen so that the average number of pixels fired per LED pulse was about 2. The output signal of the SiPM was amplified by a factor 50 using a Phillips Scientific Amplifier (Model 6954) and recorded by a CAEN charge-to-digital converter (QDC 965). The gate width was $100 \mathrm{ns,} \mathrm{and} \mathrm{the} \mathrm{start} \mathrm{of} \mathrm{the} \mathrm{SiPM} \mathrm{signal}$ was delayed by $20 \mathrm{~ns}$ relative to the start of the gate. For the absolute charge normalization we used the information from the data sheets of the different components: A gain of 50 for the amplifier and a calibration of $25 \mathrm{fC} /$ channel for the QDC 965. An overall calibration uncertainty of $10 \%$ is estimated. We note, that several of the systematic effects like cable and reflection losses, and finite integration time, result in a reduction of the measured signal. As the breakdown voltage of the $\mathrm{SiPM}$ depends on temperature, with a temperature coefficient of $56 \mathrm{mV} /{ }^{\circ} \mathrm{C}[16$, the measurements were performed in a climate chamber at a temperature of $25 \pm 0.1^{\circ} \mathrm{C}$.

Fig. 11 bottom shows the pulse-area spectrum in QDC units for a non-irradiated SiPM measured at $71.5 \mathrm{~V}$. The first peak, close to channel number 200, corresponds to zero discharging pixels, $n_{\text {pix }}=0$, with a width given by the electronics noise. The second peak corresponds to $n_{\text {pix }}=1$, with a width given by the convolution of the electronics noise and the gain distribution of the pixels. In addition, a tail is visible at the right side of the peak, which is caused by afterpulses due to charges trapped in the fired pixel and detrapped within the gate time. The following peaks correspond to $n_{\text {pix }}=2,3, \ldots 6$. The peaks of the pulse-area spectrum are individually fit by Gaussian functions, and the relation between the number of discharging pixels, $n_{\text {pix }}$, and integrated 
Table 4: Dose dependence of the gain and breakdown voltage of the different SiPMs measured at a temperature of $25.0 \pm 0.1^{\circ} \mathrm{C}$. The relative errors between the measurements are given. $\mathrm{d} G / \mathrm{d} V$ and $C_{\text {pix }}^{G}$ have an additional overall systematic uncertainty of about $10 \%$. The last column gives the change of $\mathrm{d} G / \mathrm{d} V$ relative to the $0 \mathrm{~Gy}$ value in $\%$.

\begin{tabular}{|c|c|c|c|c|c|}
\hline MPPC No. & Dose & $V_{b d}[\mathrm{~V}]$ & $\mathrm{d} G / \mathrm{d} V\left[\mathrm{~V}^{-1}\right]$ & $C_{\text {pix }}^{G}[\mathrm{fF}]$ & $\Delta[\%]$ \\
\hline \multirow{2}{*}{922} & 0 Gy & $69.40 \pm 0.03$ & $(5.50 \pm 0.04) \cdot 10^{5}$ & $88.0 \pm 0.6$ & - \\
\hline & $200 \mathrm{~Gy}$ & $69.35 \pm 0.03$ & $(5.39 \pm 0.05) \cdot 10^{5}$ & $86.2 \pm 0.8$ & -2.2 \\
\hline \multirow{3}{*}{919} & 0 Gy & $69.47 \pm 0.03$ & $(5.54 \pm 0.03) \cdot 10^{5}$ & $88.6 \pm 0.5$ & - \\
\hline & $200 \mathrm{~Gy}$ & $69.46 \pm 0.03$ & $(5.46 \pm 0.05) \cdot 10^{5}$ & $87.4 \pm 0.8$ & -1.4 \\
\hline & $20 \mathrm{kGy}$ & $69.47 \pm 0.03$ & $(5.44 \pm 0.04) \cdot 10^{5}$ & $87.0 \pm 0.6$ & -1.8 \\
\hline \multirow{2}{*}{804} & 0 Gy & $69.98 \pm 0.03$ & $(5.50 \pm 0.04) \cdot 10^{5}$ & $88.0 \pm 0.6$ & - \\
\hline & $2 \mathrm{MGy}$ & $69.93 \pm 0.03$ & $(5.27 \pm 0.06) \cdot 10^{5}$ & $84.3 \pm 1.0$ & -4.2 \\
\hline \multirow{2}{*}{805} & 0 Gy & $70.13 \pm 0.03$ & $(5.54 \pm 0.04) \cdot 10^{5}$ & $88.6 \pm 0.6$ & - \\
\hline & $2 \mathrm{MGy}$ & $70.04 \pm 0.03$ & $(5.19 \pm 0.08) \cdot 10^{5}$ & $83.0 \pm 1.1$ & -6.3 \\
\hline \multirow{2}{*}{802} & $0 \mathrm{~Gy}$ & $69.95 \pm 0.03$ & $(5.45 \pm 0.04) \cdot 10^{5}$ & $87.2 \pm 0.6$ & - \\
\hline & 20 MGy & $69.95 \pm 0.03$ & $(5.32 \pm 0.07) \cdot 10^{5}$ & $85.1 \pm 1.3$ & -2.4 \\
\hline \multirow{2}{*}{925} & 0 Gy & $69.35 \pm 0.03$ & $(5.57 \pm 0.04) \cdot 10^{5}$ & $89.1 \pm 0.6$ & - \\
\hline & 20 MGy & $69.32 \pm 0.03$ & $(5.43 \pm 0.05) \cdot 10^{5}$ & $86.9 \pm 0.8$ & -2.5 \\
\hline
\end{tabular}

charge, $Q_{\text {meas }}$, is obtained from a straight-line fit to the mean values of the Gaussian functions from the fits. The SiPM gain, $G(V)$, is calculated using

$$
G(V)=\frac{1}{q_{0}} \frac{\mathrm{d} Q_{\text {meas }}}{\mathrm{d} n_{\text {pix }}}
$$

with the elementary charge $q_{0}$. According to Eq.3 the signal from a single discharging pixel is approximately given by the product of the pixel capacitance, $C_{p i x}$, times the excess voltage, $V_{o p}-V_{b d}$, if the quench capacitance $C_{q}$ is ignored.

$$
G(V)=\frac{1}{q_{0}} C_{p i x}\left(V_{o p}-V_{b d}\right) \rightarrow C_{p i x}^{G}=q_{0} \frac{\mathrm{d} G(V)}{\mathrm{d} V}
$$

We thus expect a straight line for $G(V)$ with the slope $C_{p i x}^{G} / q_{0}$. This is actually observed, and straight-line fits are used to determine the breakdown voltage, $V_{b d}$, and the slope of the gain curve $\mathrm{d} G / \mathrm{d} V$. The errors are obtained by adjusting the uncertainties of the individual measurements so that the $\chi^{2}$ per degree of freedom is one. The results are shown in Table 4 , where also $C_{p i x}^{G}$, the pixel capacitances determined from the gain measurements, are reported. Whereas within the measurement errors the breakdown voltage does not change with irradiation, there are small but significant changes of the value of the slope: A steady decrease with dose to $\approx-5 \%$ up to a dose of $2 \mathrm{MGy}$, and a decrease by $\approx-2.5 \%$ at $20 \mathrm{MGy}$. The comparisons of the gain versus voltage measurement between 0 and $2 \mathrm{MGy}$, and between 0 and $20 \mathrm{MGy}$ for two SiPMs are shown in Fig. 12 


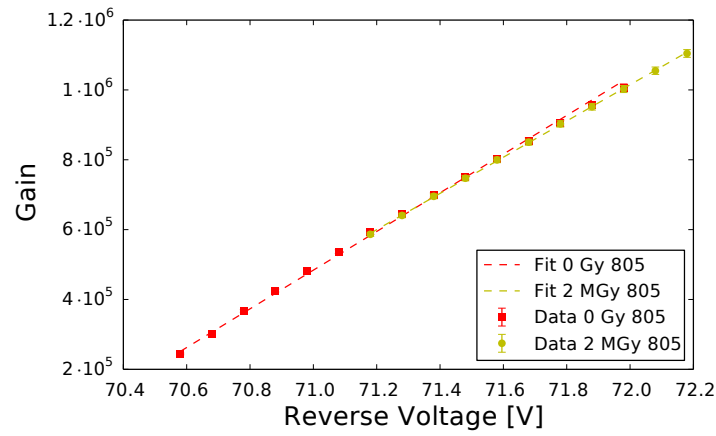

(a)

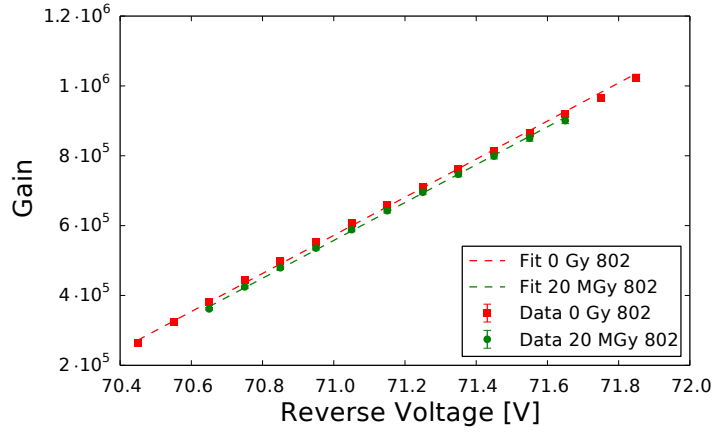

(b)

Fig. 12: Gain versus voltage measurement and straight line fit for determining the gain and breakdown voltage for (a) MPPC 805 at 0 and 2 MGy, and for (b) MPPC 802 at 0 and 20 MGy. The fit results are reported in Table 4

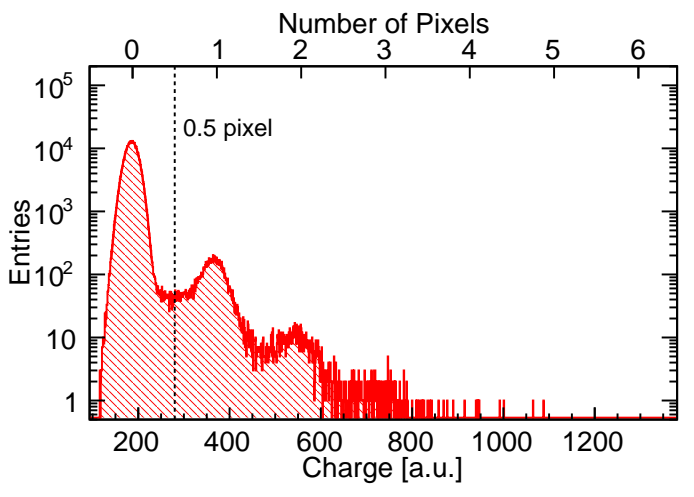

(a)

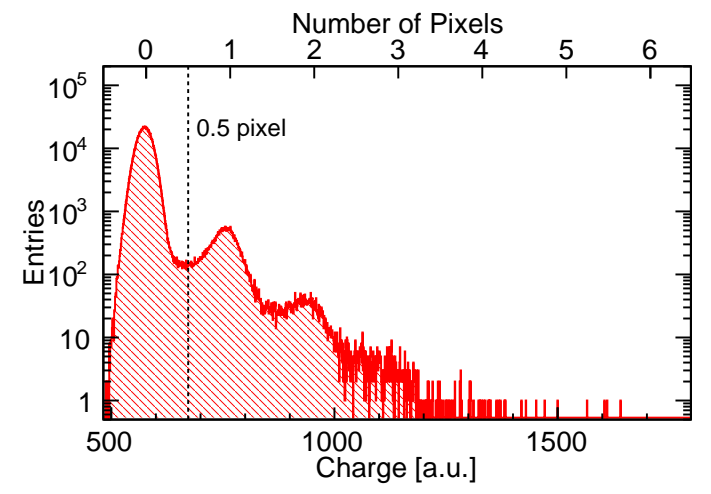

(b)

Fig. 13: Distribution of the pulse area measured in the dark at a gain of $7.5 \cdot 10^{5}$ for (a) MPPC 919 non-irradiated, and (b) MPPC 802 irradiated to 20 MGy. Counts with a pulse area above $n_{\text {pix }}=0.5$ are considered dark counts.

\subsection{Dark-count rate and dark current}

Electron-hole pairs generated in the high-field region of the SiPM, holes diffusing from the $n^{+}$substrate into the amplification region, and electrons of the surface-generation current can also trigger a Geiger discharge of a pixel and generate the same size signal as a photon. For the measurement of this dark-count rate the set-up described in Section 3.7 with the LED switched off, has been used. Fig. 13 shows the pulse-area spectrum for a non-irradiated and a SiPM irradiated to 20 MGy measured at a gain of $7.5 \cdot 10^{5}$ for a gate of length $\Delta t=100 \mathrm{~ns}$. Peaks appear at $n_{p i x}=0,1 \ldots 4$. The peaks for $n_{p i x} \geq 1$ show deviations from Gaussian functions at high values due to afterpulses within the gate time, and at low values due to noise pulses, which occur randomly in time, with only partial overlap with the gate.

The dark-count rate $\lambda$ for a given gate length $\Delta t$ is obtained from $P_{0}$, the fraction of events with pulse areas corresponding to $n_{\text {pix }}<0.5$. Assuming that the occurrence of dark counts can be described by Poisson statistics $P_{0}(\Delta t)$ is given by

$$
P_{0}(\Delta t)=e^{-\lambda \cdot \Delta t} \rightarrow \lambda=-\frac{\ln \left(P_{0}(\Delta t)\right)}{\Delta t} .
$$

This relation is only valid if the pulse length is shorter than the gate length, which is the case here, 


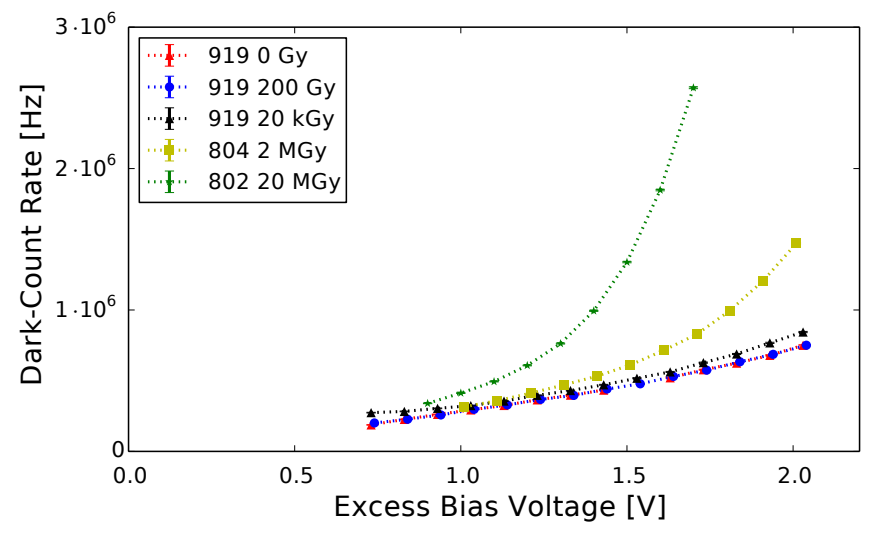

Fig. 14: Dark-count rate for several SiPMs as function of the excess bias voltage, $V_{o p}-V_{b d}$, before and after irradiation to $200 \mathrm{~Gy}, 20 \mathrm{kGy}, 2 \mathrm{MGy}$, and $20 \mathrm{MGy}$.

as shown in Section 3.10 For a precise determination of $\lambda$ the condition $P_{0} \ll 1$ should be satisfied, which is also the case. Inspection of figure 13 shows that the fraction of events with $n_{p i x}>0.5$ is much larger after the irradiation to $20 \mathrm{MGy}$ : A clear evidence for an increase of the dark-count rate due to X-ray-radiation damage.

Fig. 14 shows the dark-count rate versus excess voltage, $V_{o p}-V_{b d}$, for SiPMs before and after irradiation to $200 \mathrm{~Gy}, 20 \mathrm{kGy}, 2 \mathrm{MGy}$, and $20 \mathrm{MGy}$. Whereas the dark-count rates before and after irradiation to $200 \mathrm{~Gy}$ and $20 \mathrm{kGy}$ are similar, they increase significantly after irradiation to 2 and 20 MGy. We explain this observation by the increase of the number of electrons which reach the amplification region and cause a Geiger discharge of a pixel due to the radiation-induced increase in surface current generated at the depleted $\mathrm{Si}-\mathrm{SiO}_{2}$ interface.

\subsection{Cross Talk}

Cross talk is defined as the situation when two SiPM pixels discharge simultaneously when only one is expected. The source of cross talk are photons produced in the Geiger discharge which propagate to a neighboring pixel and produce an electron-hole pair there 11. As the propagation delay is of order femtoseconds the two signals can be considered simultaneously. In addition, there is the phenomenon of afterpulsing due to charges trapped during the Geiger discharge in the silicon bulk which detrap within the gate length of the QDC. For detrapping times shorter than the charge-up time, the pixel voltage has not yet reached the applied voltage, and the additional pulse height is smaller than the one-pixel pulse height. For longer detrapping times the additional pulse height is the normal single-pixel pulse height. The cross-talk probability is obtained from Fig. 13 as the ratio of the events above $n_{p i x}=1.5$ to the events above $n_{p i x}=0.5$ minus the probability that one or more additional noise pulses occurred within the gate width. The latter is given by $1-P_{0}$ (Eq.11). Fig. 15 shows the cross-talk probability as function of voltage before and after irradiation to $200 \mathrm{~Gy}, 20 \mathrm{kGy}, 2 \mathrm{MGy}$, and $20 \mathrm{MGy}$. The contribution from afterpulsing has not been subtracted. Whereas for small doses the cross-talk probability is essentially constant, it increases for the high dose irradiations. The reason for the increase could be that the discharges due to the dark current occur close to the pixel edges, resulting in an increased probability for photons converting in neighboring pixels. This however needs further studies.

\subsection{Pulse Shape}

For the measurement of the pulse shape the output of the $\times 50$ amplifier was digitized by a Tektronix DPO-7254 scope with $2.5 \mathrm{GHz}$ bandwidth and 20 Gsamples/second maximum sampling rate. The SiPM has been illuminated with the pulsed LED, as discussed in Section 3.7. Off-line, events with a single pixel discharging in coincidence with the LED pulse and no afterpulses have 


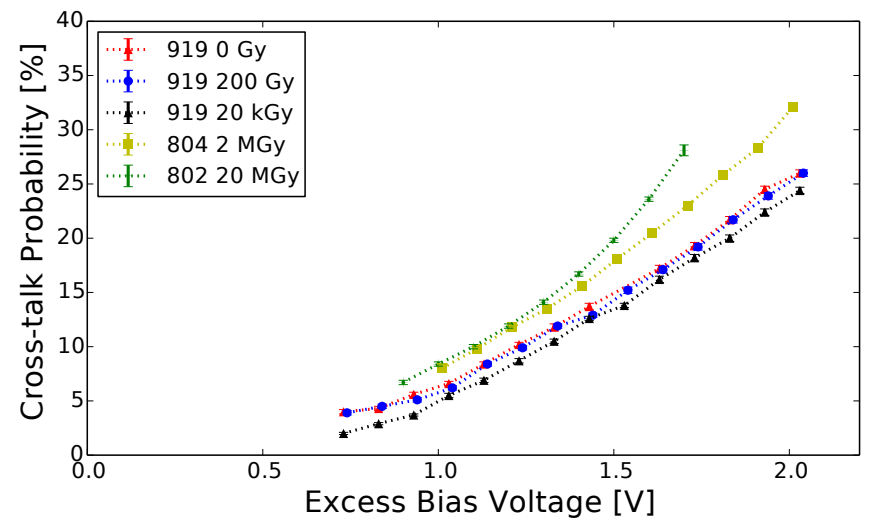

Fig. 15: Cross-talk probability for several SiPMs as function of the excess bias voltage, $V_{o p}-V_{b d}$, before and after irradiation to $200 \mathrm{~Gy}, 20 \mathrm{kGy}, 2 \mathrm{MGy}$, and $20 \mathrm{MGy}$.

Table 5: Decay time and fraction of the fast signal for SiPMs measured at a gain of $7.5 \cdot 10^{5}$ as function of dose.

\begin{tabular}{cccccc}
\hline Dose & 0 Gy & 200 Gy & 20 kGy & 2 MGy & 20 MGy \\
\hline$\tau_{d}[\mathrm{~ns}]$ & $13.6 \pm 0.5$ & $13.6 \pm 0.5$ & $13.7 \pm 0.5$ & $13.3 \pm 0.5$ & $13.8 \pm 0.5$ \\
Fraction fast signal [\%] & $5.3 \pm 0.5$ & $5.4 \pm 0.5$ & $5.2 \pm 0.5$ & $5.8 \pm 0.5$ & $5.1 \pm 0.5$ \\
\hline
\end{tabular}

been selected. The requirement was that the maximum pulse height occurred within \pm 1 ns of the time expected for the LED signal, that its value was in the range $\pm 1 \mathrm{mV}$ of $30 \mathrm{mV}$, the average of single-pixel events, and that the pulse shape did not show a step of more than $+1 \mathrm{mV}$ up to $40 \mathrm{~ns}$ after the start of the pulse.

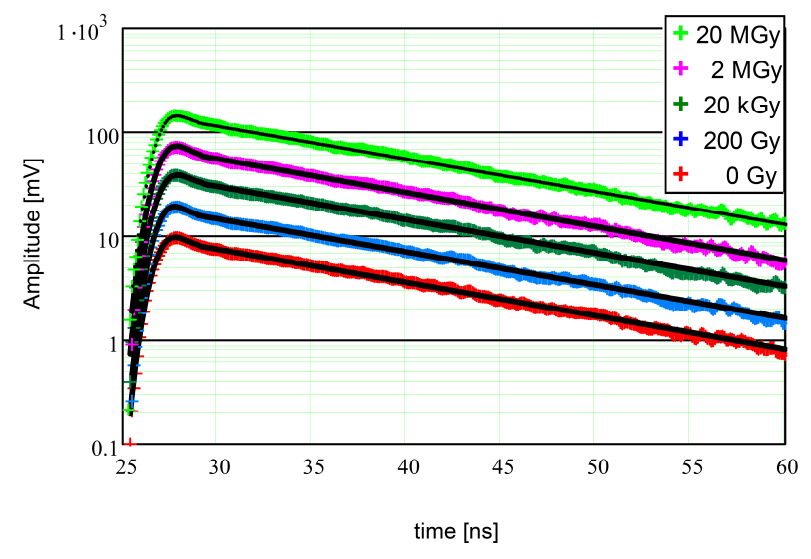

Fig. 16: Average pulse shapes of 100 single-pixel events with a selection which suppresses afterpulses, for SiPMs operated at a gain of $7.5 \cdot 10^{5}$ before and after irradiation to $200 \mathrm{~Gy}, 20 \mathrm{kGy}, 2 \mathrm{MGy}$, and 20 MGy. The solid lines show the fits described in the text. For better visibility the data are shifted by a factor two for every step in dose.

Fig.16 shows the average pulse shapes of 100 pulses thus selected before and after irradiation 
to $200 \mathrm{~Gy}, 20 \mathrm{kGy}, 2 \mathrm{MGy}$, and $20 \mathrm{MGy}$ for SiPMs operated at a gain of $(7.50 \pm 0.02) \cdot 10^{5}$. The rise time of the averaged pulses is about $1 \mathrm{~ns}$, compatible with the time jitter of the LED pulse. At the maximum there is a small peak with a width of about $1 \mathrm{~ns}$, which is related to the Geiger discharge and the value of the capacitance, $C_{q}$, in parallel to the quenching resistor, $R_{q}$ (see figure 4). The measured pulse shape has been fitted in the range between 26 and 50 ns by a $\delta$-function for the fast and an exponential for the slow component, both smeared with a Gaussian function to account for the time jitter. The results of the fit for the decay time, $\tau_{d}$, and for the contribution of the fast component to the total signal are reported in Table 5 The uncertainty has been estimated by changing the start of the fit range by $\pm 0.5 \mathrm{~ns}$, and the end of the fit range by $\pm 10 \mathrm{~ns}$. We note that, within the experimental uncertainties, decay time and fraction of fast component do not depend on X-ray dose.

Using Eq. 4 a value for the quenching capacitance of $C_{q}^{\text {pulse }}=3.3 \pm 0.3 \mathrm{fF}$ is obtained, where the value of $C_{p i x}^{C f}=93.5 \pm 1.5 \mathrm{fF}$ from Table 3 has been used for the pixel capacitance.

\section{Discussion of the results}

In this section we compare the results of the different ways used to determine the parameters of the SiPM according to the $R C$ model presented in Fig.4 and discuss their dependencies on X-ray dose.

\section{Quenching capacitance $C_{q}$}

As discussed in Section 3.5 the capacitance/conductance versus frequency measurements are not sensitive to the value of the quenching capacitance $C_{q}$. The reason is that for frequencies below $2 \mathrm{MHz}$, where the measurements were done, $\omega \cdot C_{q} \ll R_{q}^{-1}$, and the $A C$ current flows practically only through the quenching resistor $R_{q}$.

According to Eq.4 , a quenching capacitor causes a signal of pulse area $Q_{f a s t} \propto C_{q} \cdot\left(V_{o p}-V_{b d}\right)$ with a duration of about $1 \mathrm{~ns}$ or less. From the fits to the pulse shape discussed in Section 3.10 we obtain independent of the X-ray dose a value of $3.3 \pm 0.3 \mathrm{fF}$ for the quenching capacitance.

\section{Pixel capacitance $C_{p i x}$ and gain $G$}

For the pixel capacitance of the non-irradiated SiPM we determined a value of $C_{p i x}^{C f}=94.0 \pm 1.5 \mathrm{fF}$ from the capacitance-frequency measurements at $67 \mathrm{~V}$, and a value of $C_{p i x}^{G}=88 \pm 9 \mathrm{fF}$ from the gain measurements above the breakdown voltage of approximately $69.5 \mathrm{~V}$. The biggest systematic error for the comparison of the two measurements is due to the absolute calibration of the gain measurement. The decrease of $C_{p i x}$ between $67 \mathrm{~V}$ and the operating voltages is assumed to be negligible. We consider that both determinations agree within their uncertainties.

Both measurements show a decrease of $C_{p i x}$ with X-ray dose. However, the decrease from $0 \mathrm{~Gy}$ to $2 \mathrm{MGy}$ for $C_{p i x}^{C f}$ is $1.1 \%$, just at the limit of significance, whereas it is up to $\approx 5 \%$ for $C_{p i x}^{G}$. As the relative systematic error between the $C_{p i x}^{G}$ measurements is below $1 \%$, we consider the change of $C_{p i x}$ to be significant. But the change is so small and the gain of the SiPM hardly affected, so that it is not relevant for the SiPM operation.

\section{Quenching resistance $R_{q}$}

The quenching resistance has been determined in three different ways: $R_{q}^{\text {forw }}$ from the forward current, $R_{q}^{C f}$ from the capacitance/inductance versus frequency measurement, and $R_{q}^{G}$ from the decay time of the SiPM pulse. For 0 Gy the values found are: $R_{q}^{\text {forw }}=141 \pm 6 \mathrm{k} \Omega, R_{q}^{C f}=125 \pm 5 \mathrm{k} \Omega$, and $R_{q}^{G}=120 \pm 12 \mathrm{k} \Omega$. For the latter we have used Eq. 2 with $\tau_{\text {slow }}=13.5 \mathrm{~ns}, C_{q}=0, R_{L}=50 \Omega$, $C_{p i x}=94 \mathrm{fF}$, and $N_{p i x}=400$.

As discussed in Section 3.3 for the determination of $R_{q}^{\text {forw }}$ a significant extrapolation is required, with an error which is hard to estimate. We thus consider the overall agreement satisfactory. As a function of X-ray dose a decrease of $R_{q}^{C f}$ by approximately $10 \%$ between 0 Gy and 20 MGy is observed, whereas the measured decay time $\tau$, and thus $R_{q}^{G}$ remains constant within errors. We have no explanation for this difference. 
Reverse current, dark-count rate, and cross talk

Below the breakdown voltage the reverse current increases by about three orders of magnitude for $\mathrm{X}$-ray doses between 0 and $20 \mathrm{MGy}$. The increase can be explained by the radiation-induced increase in surface current from the depleted $\mathrm{Si}-\mathrm{SiO}_{2}$ interface and some charge-carrier multiplication.

Above the breakdown voltage, the situation is more complicated. Up to an X-ray dose of $20 \mathrm{kGy}$ the voltage dependence of the reverse current can be described by the sum of the dose-independent bulk current, which leads to Geiger discharges, plus the radiation-induced surface current, which is hardly amplified. The increase of the reverse current with dose is less than a factor of two for excess voltages above $0.5 \mathrm{~V}$. For X-ray doses of 2 and 20 MGy the reverse current above breakdown voltage increases by $2-3$ orders of magnitude and significant shifts of the voltage are observed, at which the current starts to increase. In addition, only part of the dark current can be explained by the measured dark-count rate, gain and cross talk. We ascribe the observed increase in reverse current to three effects: A high-field charge-carrier multiplication which does not cause Geiger discharges, $e h$ pairs generated in the bulk and $e h$ pairs at the $\mathrm{Si}-\mathrm{SiO}_{2}$ interface, which both cause Geiger discharges. High-field breakdown critically depends on environmental parameters like humidity [29, which has not been controlled for the measurements reported here. Electrons generated at the $\mathrm{Si}-\mathrm{SiO}_{2}$ interface are responsible for the observed dose dependence of the dark-count rate, $D C R$, on X-ray dose: An X-ray dose of $200 \mathrm{~Gy}$ does not affect the $D C R$, at $20 \mathrm{kGy}$ a small but significant increase at the $10 \%$ level is observed, and finally at $20 \mathrm{MGy}$ the $D C R$ increases by about an order of magnitude for excess voltages above $1.8 \mathrm{~V}$.

The X-ray dose also affects the cross-talk probability, however in a minor way. Up to a dose of $20 \mathrm{kGy}$ there is practically no effect, for higher doses an increase by up to $50 \%$ is observed.

\section{Conclusions and Outlook}

The paper presents a detailed characterization of the SiPM MPPC S10362-11-050 Crom Hamamatsu below and above breakdown voltage, as well as for forward biasing, before and after irradiation with X-rays to $200 \mathrm{~Gy}, 20 \mathrm{kGy}, 2 \mathrm{MGy}$, and $20 \mathrm{MGy}$ without applied voltage. The measurements performed after the irradiations were: current-voltage, capacitance/conductancevoltage for frequencies between $100 \mathrm{~Hz}$ and $2 \mathrm{MHz}$ below the breakdown voltage, and gain, dark-count rate, cross-talk probability and pulse shape above the breakdown voltage.

The data allows us to determine characteristic parameters of the SiPM, such as quench resistor, pixel capacitance, dark-count rate and pulse shape, as a function of X-ray dose in different ways. The parameters determined in different ways generally agree within their estimated uncertainties. In addition, the doping profile and electric field of the SiPM has been extracted from the measurements.

As a function of X-ray dose, changes of values of several parameters are observed, in particular below breakdown voltage the dark current, and above breakdown voltage dark-count rate and cross-talk probability. The study shows that the MPPC S10362-11-050 C from Hamamatsu can be operated after X-ray irradiation to a dose of $20 \mathrm{MGy}$. Up to $20 \mathrm{kGy}$ the changes are minor, whereas for a dose value of 20 MGy the dark-count rate increases by an order of magnitude.

The study presented is a first step by the Hamburg group towards a systematic investigation of radiation effects on SiPMs. With respect to X-ray-radiation damage the planned next steps are to study the sensor performance during and shortly after X-ray irradiation, where large pulses and currents have been observed previously, investigate the influence of environmental conditions like humidity, and extend the study to SiPMs from different producers.

\section{Acknowledgment}

The authors would like to thank S. Schuwalow who derived the analytic solutions of the equations of the pulse-shape model, J.Zhang who helped with the X-ray irradiation, E. Popova, M. Ramilli, J. Schwandt and A. Silenzi for fruitful discussions, and P. Buhmann, W. Gärtner and M. Matysek for the continuous improvement and maintenance of the measurement infrastructure of the laboratory and for helping in the measurements. Chen $\mathrm{Xu}$ has received funding from the 
European Union Seventh Framework Programme (FP7/2007 - 2013) under Grant Agreement no. 256984 (Endo-TOFPET-US).

\section{List of References}

\section{References}

[1] D. Renker and E. Lorenz, Journal of Instrumentation 4 (2008) P04004, doi: 10.1088/1748-0221/4/04/P04004.

[2] J. Haba, Nuclear Instruments and Methods in Physics Research Section A 595 (2008) 154-260, doi: 10.1016/j.nima.2008.07.061.

[3] P. Buzhan et al., Nuclear Instruments and Methods in Physics Research Section A 504 (2003) 48-52, doi: 10.1016/S0168-9002(03)00749-6.

[4] Y. Musienko et al., Nuclear Instruments and Methods in Physics Research Section A 581 (2007) 433-437, doi: 10.1016/j.nima.2007.08.021.

[5] I. Nakamura, Nuclear Instruments and Methods in Physics Research A 610 (2009) 110-113, doi: 10.1016/j.nima.2009.05.086.

[6] T. Matsubara, H. Tanaka, K. Nitta, and M. Kuze, 2007 POS (PD07) 032.

[7] S. Sánchez Majos et al., Nuclear Instruments and Methods in Physics Research Section A 602 (2009) 506-510, doi: 10.1016/j.nima.2009.01.176.

[8] Y. Qiang, C. Zorn, F. Barbosa, and E. Smith, Nuclear Instruments and Methods in Physics Research Section A 698 (2013) 234-241, doi:10.1016/j.nima.2012.10.015.

[9] W.L. Hellweg, Radiation Damage on Hamamatsu S 10943 Silicon Photomultipliers, BSC thesis, University of Hamburg, September 2013.

[10] C.Xu, Study of the Silicon Photomultipliers and Their Applications in Positron Emission Tomography, PhD thesis, University of Hamburg, April 2014.

[11] T.R. Oldham, Ionizing Radiation effects in MOS Oxides, World Scientific Publishing Co. (1999).

[12] H.J. Barnaby, IEEE Trans. Nucl. Sci. 53 (2006) 3103.

[13] R. Klanner et al., Nuclear Instruments and Methods in Physics Research Section A 732 (2013) 117-121, doi:10.1016/j.nima.2013.05.131.

[14] J. Zhang et al., 2012 JINST 7 C12012, doi:10.1088/1748-0221/7/12/C1201.

[15] J.Zhang, X-ray radiation damage studies and design of a silicon pixel sensor for science at the XFEL, PhD thesis, University of Hamburg, DESY-THESIS-2013-018, June 2013.

[16] Hamamatsu data sheet for MPPCs (multi-pixel photon counters): http://www.hamamatsu.com/us/en/4004 html

[17] http://petra3.desy.de/index_eng.html

[18] K. Yamamoto et al., 2007 POS (PD07) 004.

[19] O. Marinov, M. Jamal Deen, and J. A. Jimenez Tejade, J. Appl. Phys. 101 (2007) 064515, doi:10.1063/1.2654973.

[20] S. Cova et al., Applied Optics, 35(12):1956-1976, 1996.

[21] F. Corsi et al., Nuclear Instruments and Methods in Physics Research Section A 572 (2007) 416-418, doi:10.1016/j.nima.2006.10.219.

[22] Wei Chen, Development of High Performance Readout ASICs for Silicon Photomultipliers (SiPMs), PhD thesis, University of Heidelberg, 2012.

[23] A.D. Pleshko, P.Zh. Buzhan, A.I. Il'in, et al., Studying Voltage Recovery Processes on Silicon Photomultiplier Tubes, Priboryi Tekhnika Eksperimenta (2013) No.6, pp.60-68 (in Russian), English translation in Instruments and Experimental Techniques (2013), Vol. 56, No. 669-677, Pleiades Publishing, Ltd., 2013, ISSN 00204412.

[24] D. M. Fleetwood, IEEE Transaction of Nuclear Science Vol. 39, No. 2 (1992).

[25] A.S. Grove, Physics and Technology of Semiconductor Devices, John Wiley \& Sons (1967).

[26] D.K. Schroder, Semiconductor Material and Device Characterization, John Wiley \& Sons (2006).

[27] G. Kirchhoff, Zur Theorie des Condensators, Gesammtsitzung der Akademie der Wissenschaften zu Berlin vom 15. März 1877.

[28] P. Eckert et al., Nuclear Instruments and Methods in Physics Research Section A 620 (2010) 217-226, doi: 10.1016/j.nima.2010.03.169.

[29] F. G. Hartjes Nuclear Instruments and Methods in Physics Research Section A 552 (2005) 168-175, doi: 10.1016/j.nima.2005.06.027. 